\title{
1 Mapping Mind-Brain Development: Towards a 2 Comprehensive Theory
}

3 George Spanoudis ${ }^{1, *}$ and Andreas Demetriou ${ }^{2}$

1 Psychology Department, University of Cyprus 2; spanoud@ucy.ac.cy

2 University of Nicosia 1; ademetriou@ucy.ac.ac.cy

* Correspondence: spanoud@ucy.ac.cy Tel.: +35722892969)

Received: date; Accepted: date; Published: date

Abstract: The relations between developing mind and developing brain are explored. We outline a theory of intellectual development postulating that the mind comprises four systems of processes (domain-specific, attention and working memory, reasoning, and cognizance) developing in four cycles (episodic, realistic, rule-based, and principle-based representations, emerging at birth, 2, 6, and 11 years, respectively), with two phases in each. Changes in reasoning relate with processing efficiency in the first and working memory in the second phase. Awareness of mental processes recycles with them in each cycle and drives their integration into the representational unit of the next cycle. Brain research shows that each type of processes is served by specialized brain networks. Domain-specific processes are rooted in sensory cortices; working memory processes are mainly rooted in hippocampal, parietal, and prefrontal cortices; abstraction and alignment processes are rooted in parietal, frontal, and prefrontal and medial cortices. Information entering these networks is available to awareness. Brain networks change along the four cycles, in precision, connectivity, and brain rhythms. Principles of mind-brain interaction are discussed.

Keywords: Brain 1; Mind 2; Development 3; Mental and Brain Changes 4; Brain Networks 5.

\section{Introduction}

The brain is a biophysical system collecting physical messages from the environment with self as the reference system. It uses this information to construct a representation of the environment to guide action by its bearer. Representations, ensuing understanding, action planning, and the psychological mechanisms involved comprise the mind. Therefore, the brain is the biological substrate of the mind, because the mind emerges, in all of its expressions, from the structure and functioning of the brain. In this paper we examine how these levels of analyzing mental functioning, the psychological and the biological, relate with each other. The frame for discussion is current research and theory on the organization, functioning, and development of (i) intellectual processes and (ii) the human brain. The paper is organized in two parts: the first focuses on the architecture and functioning and the second on the development of mind and brain. In each of these parts we first outline the basic findings and principles about the mind and then present evidence and theory about the underlying brain structures and mechanisms. The paper aims to answer the following four questions:

1. Is the architecture of mind suggested by psychological research reflected in the organization and functioning of the brain?

2. What are the neuronal processes implementing mental processes and mechanisms, such as abstraction and reasoning?

3. How are developmental changes in gross parameters of the brain (such as increases in neuronal volume, myelination, networking, and pruning) reflected in developmental changes of cognitive processes?

4. Are there systematic individual differences in brain architecture and functioning that may be connected to individual differences in intellectual functioning and development? 
We caution that answering these questions is not an easy matter. On the one hand, psychological research involves: (i) observable responses expressed in different scales and (ii) subjective experiences expressed in various modes, such as self-descriptions, self-evaluations, certainty evaluations, etc. On the other hand, brain research involves biological entities, such as the nature, volume, and organization of neuronal matter and its functional correlates, such as brain rhythms, expressed through various modes (e.g., blood supply and glucose consumption of activated brain areas and electrochemical activity). Structurally, the human brain comprises a large number of cytoarchitecturally discrete regions, such as Brodmann's (1909) areas or more detailed maps identified by recent research (Glasser et al. 2016). Despite the diversity of brain regions, the brain operates as a single integrated entity governed by multiple processes running within a highly constrained, genetically organized, context interacting with the environment (Stiles and Jernigan 2010). The scales and precision of measuring each of these dimensions vary both within and across the two levels of description. Thus, we often do not know how to map the different levels of analysis onto each other and when we map them precision falls short of the optimal. Martínez et al. (2015) recently demonstrated dramatically that the relations between different aspects of cognition, such as fluid intelligence and working memory, cannot be consistently mapped on the same brain dimensions, when the methods of brain mapping vary. With these reservations granted, we will attempt to set a tentative frame for bridging the two fields.

\section{Architecture}

The human mind is a hierarchical universe involving multiple systems at different levels of organization. Systems at different levels involve processes serving different tasks during understanding and problem solving. Some are domain-specific, dealing with particular types of information and relations in the environment and some are general, dealing with the relations between mental entities and the production of new ones. Overall, cognitive and psychometric research (Demetriou and Spanoudis 2018) suggests four types of systems: domain-specific, attention and memory, integrative, and cognizance systems. Figure 1 illustrates this architecture in the conventions of structural equation modeling. It can be seen that domain-specific systems are related to a common factor, g; in turn, this factor is strongly related with markers of domain-general representational (i.e., attention and working memory), integrative (i.e., reasoning), and self-awareness (i.e., cognizance) processes. This model was tested in several studies presented elsewhere (Demetriou and Spanoudis, 2018; Demetriou et al., 2002, 2017, 2018; Makris et al., 2017). Below we focus on each type of systems, outlining its psychological profile first and then discussing relevant brain research. To facilitate the reader to map the psychological architecture of the mind onto research about the architecture of the brain, we organize the text in two sections for each major aspect of the architecture, one for the mind and one for the brain.

\subsubsection{Domain-Specific Mental Processes}

Domain-specific systems interface the mind with different aspects of the environment. Psychometric, cognitive, and developmental research agree on five domains of thought: verbal, quantitative, spatial, causal, social, and lingustic. All domains emerge as distinct dimensions in various types of modelling performance on cognitive tasks such as exploratory and confirmatory factor analysis, and graph analysis (see Figure 1). These domains have been described and substantiated elsewhere (Carey 2009; Carroll 1993; Case 1992; Demetriou et al. 1993; Case et al. 2001; Demetriou and Bakrasevic 2009; Demetriou et al., 1993, 2002; Demetriou and Kazi, 2006; Gelman 2003; Golino and Demetriou 2017). They will only be outlined here for the present purposes.

Each domain involves: (i) perception-based core processes and (ii) representation-based mental operations. Table 1 summarizes the composition of domains across these two types of processes. Core processes are rooted in perception. They frame how meaning is initially made of objects and they appear early in life, if not at birth. Color perception in categorical thought (Eguchi et al. 2017), subitizing in quantitative thought (i.e., automatic recognition of the numerosity of small sets) (Dehaene 2011), depth perception in spatial thought (Hermer and Spelke 1996), perception of causality in causal thought (Saxe and Carey 2006), and face perception in social thought (Simion et al. 2001) are present around birth to ignite the construction of mental operations and concepts. With development, core processes 


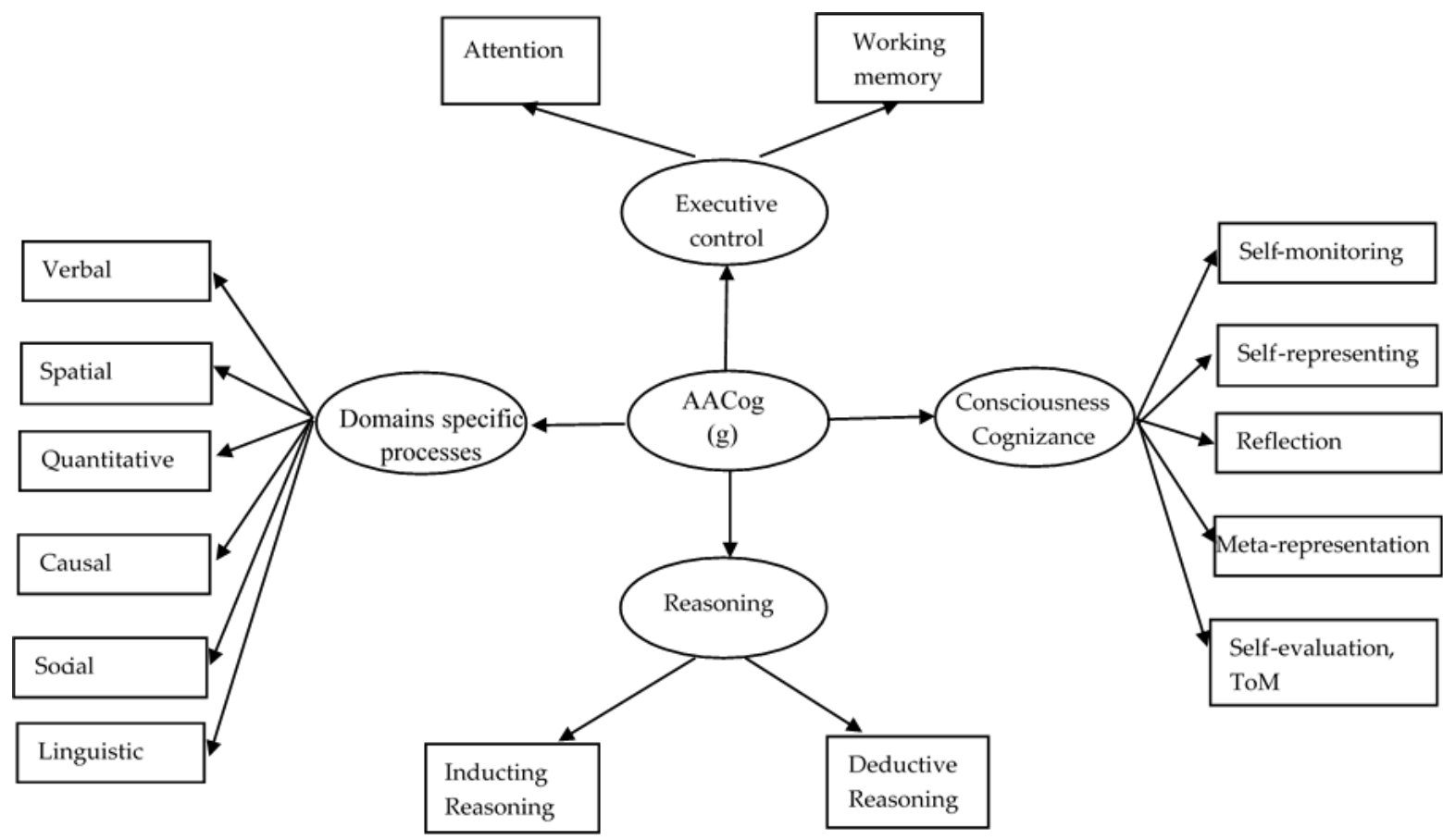

Figure 1. The cognitive architecture of the mind.

Note: AACog stands for Abstraction, Alignment, and Cognizance.

develop into domain-specific operations, such as sorting in categorical reasoning, mental rotation in spatial reasoning, arithmetic operations in quantitative reasoning, isolation of variables in causal reasoning, and moral reasoning in social thought (Demetriou and Spanoudis 2018). Basically, domainspecific operations are generalizations of core processes. They include systems of actions and representations integrating variations in the functioning of core processes over occasions and they may fill in lags of information when core processes do not suffice. The functioning of core processes and operations generates knowledge and beliefs about the domains concerned.

Table 1: The three levels of organization of each specialized system of thought.

\begin{tabular}{llll}
\hline Domain & \multicolumn{1}{c}{ Core Processes } & \multicolumn{1}{c}{ Mental Operations } & $\begin{array}{l}\text { Knowledge and } \\
\text { Beliefs }\end{array}$ \\
\hline Verbal & $\begin{array}{l}\text { Perception according to } \\
\text { perceptual similarity; inductive } \\
\text { inferences based on similarity- } \\
\text { difference relations }\end{array}$ & $\begin{array}{l}\text { Specification of the semantic } \\
\text { and logical relations between } \\
\text { properties, classification; } \\
\text { transformation of properties } \\
\text { into mental objects; } \\
\text { construction of conceptual } \\
\text { systems }\end{array}$ & $\begin{array}{l}\text { Conceptions } \\
\text { and } \\
\text { misconceptions } \\
\text { about the world }\end{array}$ \\
Quantitative & $\begin{array}{l}\text { Monitoring, reconstruction, } \\
\text { execution and control of } \\
\text { pointing, bringing in, } \\
\text { removing, sharing }\end{array}$ & $\begin{array}{l}\text { Factual } \\
\text { the four arithmetic operations } \\
\text { knowledge } \\
\text { about the }\end{array}$ \\
& & & $\begin{array}{l}\text { quantitative } \\
\text { aspects of the } \\
\text { world, algebraic } \\
\text { and statistical } \\
\text { inference rules }\end{array}$
\end{tabular}




\begin{tabular}{|c|c|c|c|}
\hline Spatial & $\begin{array}{l}\text { Perception of size, depth, and } \\
\text { orientation; formation of } \\
\text { mental images }\end{array}$ & $\begin{array}{l}\text { Mental rotation, image } \\
\text { integration, image } \\
\text { reconstruction, location and } \\
\text { direction tracking and } \\
\text { reckoning }\end{array}$ & $\begin{array}{l}\text { Stored mental } \\
\text { images, mental } \\
\text { maps, and } \\
\text { scripts about } \\
\text { objects, } \\
\text { locations, } \\
\text { scenes, or } \\
\text { layouts } \\
\text { maintained in } \\
\text { the mind }\end{array}$ \\
\hline Causal & $\begin{array}{l}\text { Perception of overt and covert } \\
\text { causal relations }\end{array}$ & $\begin{array}{l}\text { Trial and error; combinatorial } \\
\text { operations; hypothesis } \\
\text { formation; systematic } \\
\text { experimentation (isolation of } \\
\text { variables); model construction }\end{array}$ & $\begin{array}{l}\text { Knowledge, } \\
\text { attributions and } \\
\text { understanding } \\
\text { of the reasons } \\
\text { underlying } \\
\text { physical and } \\
\text { social events } \\
\text { and the } \\
\text { dynamic aspects } \\
\text { of the world }\end{array}$ \\
\hline Social & $\begin{array}{l}\text { Recognition of conspecifics, } \\
\text { recognition of emotionally } \\
\text { laden facial expressions }\end{array}$ & $\begin{array}{l}\text { Deciphering the mental and } \\
\text { emotional states and } \\
\text { intentions of others; } \\
\text { organization of actions } \\
\text { accordingly; imitation; } \\
\text { decentering and taking the } \\
\text { other's perspective }\end{array}$ & $\begin{array}{l}\text { System of social } \\
\text { attributions } \\
\text { about other } \\
\text { persons, their } \\
\text { culture and their } \\
\text { society }\end{array}$ \\
\hline Linguistic & $\begin{array}{l}\text { Use of the grammatical and } \\
\text { syntactical structures of } \\
\text { language }\end{array}$ & $\begin{array}{l}\text { Identifying truth in } \\
\text { information; abstraction of } \\
\text { information in goal-relevant } \\
\text { ways; differentiation of the } \\
\text { contextual from the formal } \\
\text { elements; elimination of } \\
\text { biases from inferential } \\
\text { process; securing validity of } \\
\text { inference }\end{array}$ & $\begin{array}{l}\text { Knowledge } \\
\text { about grammar, } \\
\text { syntax and } \\
\text { logical } \\
\text { reasoning; } \\
\text { metalogical } \\
\text { knowledge } \\
\text { about nature } \\
\text { and justifiability } \\
\text { of logical } \\
\text { inferences; } \\
\text { metacognitive } \\
\text { awareness, } \\
\text { knowledge, and } \\
\text { control of } \\
\text { inferential } \\
\text { processes }\end{array}$ \\
\hline
\end{tabular}

110 The first question in the introduction asked if the architecture of mind suggested by psychological 111 research is reflected in the organization and functioning of the brain. This seems to be the case when 112 we come to core processes and domain-specific operations. Research suggests that there are entry nodes 113 in the sensory cortices corresponding to each of the core operations above which are highly modular 
(Yue et al. 2017). Specifically, corticocortical networks display modular organization, alternative processing paths, and highly connected hubs (Zamora-López et al. 2010). Different aspects of visuospatial information, such as depth and orientation or gloss, which stand for different core processes in spatial thought, are processed by overlapping regions in the visual cortices (dorsal visual area V3B/KO; kinetic occipital region; Dövencioğlu et al. 2013; Livingstone and Hubel, 1988; Sun et al. 2015). Discrete quantitative information, such as subitization, and separate numbers are processed by specific brain networks located in the visual and the parietal cortex (Dehaene, 2011; Anobile et al. 2015). Perception of causality is served by visual cortices, such as V5 (Rolfs et al. 2013). These cortices, in the right hemisphere, register the actual physical relations between causally interacting objects, abstracting causality from rapid interactions between moving objects (Roser et al. 2005). Finally, in social understanding, the inferior occipital gyrus seems to be the entry node for face recognition (Zhen et al. 2013). Figure 2 illustrates the main regions associated with each domain.

Information from entry nodes is sent to more general purpose integrative cortices that may be associated with mental operations (Taylor et al., 2015; Yue et al., 2017). Specifically, relational processing underlying mental operations is carried forward, reaching the associative parietal and integrative frontal cortices and temporal cortices, when language is involved. Processing of verbally expressed categorical information is associated with the entire superior temporal gyrus, which is related to language understanding and it thus analyzes the "object" properties of auditory signals (Galaburda et al. 2002). Implementing already acquired expertise in object categorization, regardless of their identity, involves the fusiform gyrus in the inferior extrastriate visual cortex (Gauthier et al. 2000). Mental rotation, an integrative operation of spatial thought, activates the right posterior parietal lobe, centered on the intraparietal sulcus (B7; it is noted that there are inconsistencies in the literature in naming brain areas; some researchers use the Brodmann system for naming areas in the cortex and others use anatomical terms specifying brain areas in 3-dimensional space; in this paper we kept the original naming system of each article to avoid misspecification of areas; however, when the Brodmann system is used we also use the anatomical terms in parenthesis to minimize confusion); three-dimensional rotation also activates the right dorsal premotor cortex. (Harris et al. 2000; Kawamichi et al. 2007). Categorical encoding of spatial positions activates more the parietal cortex within the left hemisphere than within the right. However, encoding of the same relations in spatial coordinate terms caused more activity in medial temporal cortex and dorsal striatum (Baumann et al. 2012). Number relations, such as increase along the number line, are processed in the inferior parietal cortex, the angular gyrus (BA 39) in particular, and the intraparietal sulcus. Notably, the horizontal segment of the intraparietal sulcus (hIPS) seems to serve the symbolic representation of number by interlinking neural populations directly indicating number, such as dots, with neural populations coding for number symbols, such as digits and number words (Piazza et al. 2007). The frontal cortex is also involved (Dehaene 2011; Nieder and Dehaene 2009). Inference about causal relations is associated with activity in the left hemisphere, where causality from contingencies between events is inferred, such as first pressing a switch and then observing its effect, i.e., the onset of a light (Fonlupt 2003; Roser et al. 2005). Finally, face encoding involves, in addition to occipital regions, right hippocampus and left and right prefrontal cortex, showing similarities with expert categorization (Haxby et al. 1996; Kanwisher et al. 1997; Zhen et al. 2013).

\subsubsection{Mental Systems for Attention and Working Memory}

There is general agreement that a complex attention system enables the organism to focus on stimuli of importance according to a goal and control interference from surrounding irrelevant but prominent stimuli. The attention system involves three fundamental components: an alerting system controlling arousal and vigilance; an orienting system related to the prioritization of input through selection of modality or location; and a top-down system that handles conflicts and is related to executive control. The current discussion about mastering executive control in development basically refers to the acquisition of self-directed actions that may enable the individual to subject the three attention components under intentional control (Zelazo, 2015).

Attention systems deliver their content to retention processes operating in the sake of integration of current information with past experience, current action, and planning. There are three types of 
retention processes: (i) modality-specific short-term storage processes (STS) related to the perceptual origin of information, such as acoustic and visual storage; (ii) executive processes, representing the currently active mental goal controlling information search and integration; together, these two types of processes are known as working memory; (iii) an episodic integrator capable of integrating information across modes into unitary episodic representations preserving time and subjectivity of experience (Conway 2005). Strictly speaking, of these processes, only STS directly serves retention. Executive and episodic integration processes may actually belong to the other types of systems, such as integrative and awareness systems to be discussed below. Brain research may resolve this possible confounding (Baddeley 2012; Cowan 2010; Pennartz 2015).

\subsubsection{Brain Systems for Attention and Working Memory}

2.2.2.1 Attention. Each of the mental attention systems above is served by a different network in the brain (Posner and Rothbart 2007). The alerting system is based in the brain stem system and the thalamus. This is a bottom-up network that projects to the frontal and the parietal cortices. The activation of this network renders one ready for awareness but it does not by itself generate awareness (Dehaene et al. 2017). The orienting system is served by the frontal eye fields (FEF) and parietal areas and frontal areas. Moreover, this system seems to involve two discrete functions, one serving top-down visuospatial selection [intra-parietal sulcus (IPS)/superior parietal lobule (SPL) and FEF] and one serving bottom-up reorienting [temporoparietal junction (TPJ), i.e., inferior parietal lobule (IPL)/superior temporal gyrus (STG) and ventral frontal cortex (VFC), i.e., inferior frontal gyrus (IFg)/middle frontal gyrus (MFg)]. This system may be the gate to conscious awareness discussed below. Finally, the inhibition and executive system involves two networks, a frontoparietal network (precuneus, middle cingulate cortex (mCC), dorsolateral prefrontal cortices (dlPFC), dorsal frontal cortices (dFC), IPS, and IPL) serving moment-to-moment task monitoring; the second is a cinguloopercular network: dorsal anterior cingulate cortex (dACC)/medial superior frontal cortex (msFC), anterior prefrontal cortex ( $\mathrm{aPFC})$, anterior insula/frotal operculum $(\mathrm{al} / \mathrm{fO})]$ serving task maintenance. This system may yield awareness of the objects of current thought (Dehaene et al. 2017) (see Figure 2).

2.2.2.2 Working memory processes. Several brain structures and networks serve working memory. Jonides et al. (2005) suggested that STS is mediated by the same structures that process perceptual information. That is, when perceptually activated, information-specific brain modules retain information and relay it for further processing to a higher-level system that integrates over event sequences within the same module or across modules, such as integration of visual and acoustic information: phonological storage is associated with a bilateral anterior-prefrontal/inferior parietal network; visuo-spatial storage is associated with posterior parts of the superior frontal sulcus and the entire intraparietal sulcus. Some brain regions, such as the frontoparietal systems associated with attention are activated during processing of both, verbal rehearsal and the visuo-spatial transformations, such as spatial sequencing and rotation (Baddeley 2012; Gruber and Cramon 2003; Repovš and Baddeley 2006).

Hippocampus has a special role in episodic integration, specializing in the explicit representation of novel information and temporal and spatial order. It temporarily binds together several distributed areas in the cortex representing various kinds of information (Conway 2005; Meeter et al. 2005; Sun et al. 2020). In fact, it is itself differentiated so that different cell assemblies in it serve different aspects of working memory, such as (i) retaining information received from visual or phonological cortices, (ii) specifying arrangement information, such as object location and sequential order (Lisman 2005), and (iii) delivering information to the frontal cortex for relational integration (Friedman and Goldman-Rakic 1988; Rubin et al. 2017).

These retention networks interact with attention networks that may ensure attention control in its various manifestations: focus on and maintain the currently relevant items (e.g., ventral and/or posterior prefrontal cortex (PFC) maintains the currently relevant items (Ranganath and D' Esposito 2005); construct multimodal object representations (e.g., medial temporal and inferior temporal regions); and shift between objects, if needed i.e., the inferior frontal junction (IFJ). This later function causes the attentional bottleneck because it can retain only one rule at a time (Vergauwe et al. 2015). 
Finally, response selection correlates with activation in medial and ventrolateral pre-frontal regions (Cowey 1996; Gruber and Goschke 2004; Rubin et al. 2017; Yoon et al. 2008). When selection is effected processing is delivered to the integrative and awareness processes discussed below. These findings indicate that executive processes belong to integrative or awareness systems rather than to retention as such.
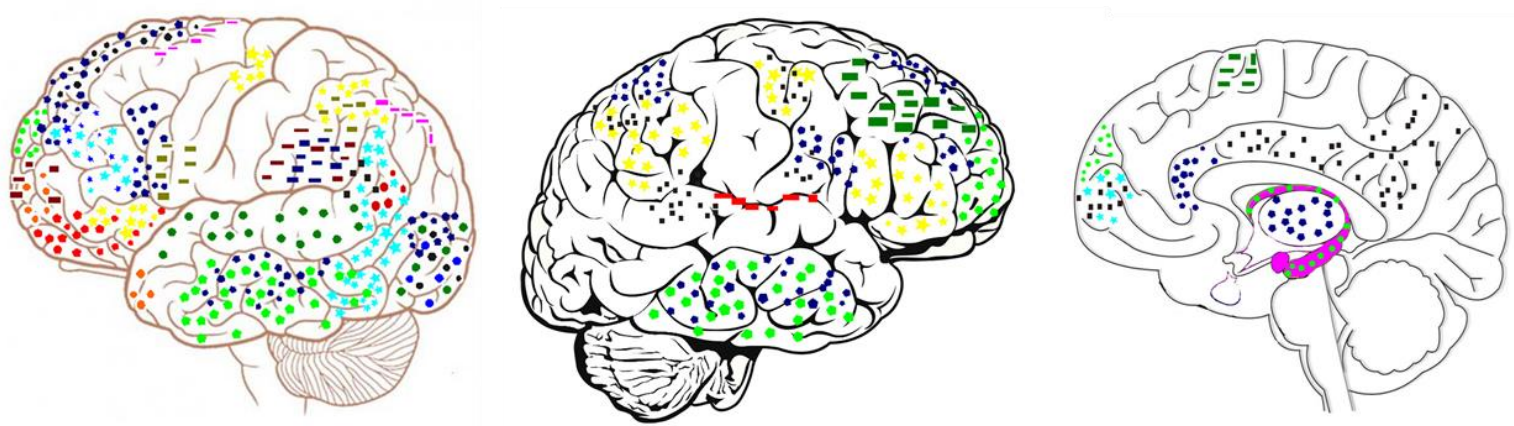

Figure 2. Brain regions associated with different mental processes. Note: Shape indicates systems of processes and color indicates specific processes within a system.

\begin{tabular}{|c|c|c|c|c|c|c|c|c|c|}
\hline Domain-specific & 0 & Working memory & $\square$ & Attention & 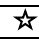 & Inferential reasoning & 0 & Cognizance & $\square$ \\
\hline Verbal & & Visual & & Inhibition & & Binding & & Theory of mind & \\
\hline Quantitative & & Verbal, reheasral & & Selection & & Deductive & & & \\
\hline Spatial & & Verbal, maintenance & - & Switching & & Inductive & & & \\
\hline Causal & & Episodic & & & & & & & \\
\hline Social & & & & & & & & & \\
\hline
\end{tabular}

\subsubsection{Integrative Mental Processes}

In psychometric theory, general intelligence, $\mathrm{g}$, stands for these processes which are thought to significantly account for individual differences on any cognitive task. It is beyond the scope of this article to embark on the long-standing debate about the nature of $\mathrm{g}$. Interpretations vary enormously. Inductive and analogical reasoning (Spearman 1904; Gustafsson 1984), processing speed (Jensen 1998), working memory (Cowan 2010; Kyllonen and Christal 1990), and executive control (Blair 2006) were invoked as the core of $g$. However, none of these processes correlates with $g$ higher than .5 , indicating that $\mathrm{g}$ cannot be reduced to any one of them. In response to this state of affairs, several scholars stripped $\mathrm{g}$ of any distinct psychological process, claiming that $\mathrm{g}$ is an algebraic consequence of the interaction between specific processes (van der Maas et al. 2017), perhaps with some of them being more powerful than others in driving the interaction, such as executive attention that is shared by most cognitive tasks (Kovacs and Conway 2016). Our research showed that all of these processes, together with awareness, are additive components of $\mathrm{g}$, capturing 98\% of variance in any domain (Demetriou et al. 2017, 2018 ; Makris et al. 2017) (see Figure 1).

The common core of the mind must be general enough to cut across all four types of systems (domain-specific, attention and retention, inferential, and awareness). This core must have indentifiable properties at both the psychological level and the neuronal level going beyond the specifics in each of the four types of processes. In sake of this aim, we suggest a mechanism that may look for and extract patterns from information or representations, inter-relate them and generate new patterns or representations that may be more inclusive and accurate in representing the situations of interest. This mechanism involves three inter-dependent processes: (i) representational Alignment; (ii) Abstraction; and (iii) Cognizance. This is the AACog mechanism. Alignment is a "search, vary, and compare" relational mechanism interlinking stimuli and/or representations together according to current goals; thus, alignment is an executive mechanism of representational integration, involving shifting between representations or between representations and responses (Miyake and Friedman 2012). Abstraction spots or induces similarities between patterns of information based on perceptual or semantic properties which feed further alignment processes. Cognizance is part of reflexive consciousness (Dehaene, Lau, \& Kouider, 2017), which includes awareness of one's own mental processes ("I know that I see by my eyes"), knowledge ("I know I saw apples before"), and abilities ("I can remember what 
I saw before"). It draws on self-monitoring, reflection, and metarepresentation, allowing explicit encoding of abstractions and reflections on them into new representations (see Figure 1).

To function, AACog, requires attention and working memory processes but it goes beyond them in that it processes the contents of attention and memory. Cognizance is required because it extends experience beyond the here and now. It may arise any time automatic pattern abstraction is not possible or alignment goes wrong. Choosing between stimuli or actions turns the "mind's eye" to them thereby bringing them into the focus of awareness. Thus, cognizance brings a particular mental content in focus and shifting between mental contents. So defined cognizance allows feedback loops where cycles of alignment of representations, abstraction of relations, and inference may become the object of further alignment and abstraction until a final conclusion is reached, often projected into a new concept. From an adaptational point of view, cognizance reflects the mind's need to re-work experiences in order to select among them, stabilize whatever appropriate, and integrate with past experience. Thus, it is the basis of mental time-line where past, present, and future are aligned.

Cognizance processs may bring specific mental processes themselves in focus. For instance, trying to align stimuli may bring memory into focus (because some information is lost in the process); abstracting relations may bring inference in focus. Metarepresentation is a fundamental component of cognizance in that it encapsulates the results of abstraction and alignment into new mental units and encodes them into new representations that may be retrieved as such (e.g., a new word, a new personal mental image, or a new arbitrary symbol). A series of studies suggested that cognizance is an integral component of general intelligence (Demetriou and Kazi 2006), it recycles in development with the cognitive processes dominating in each cycle (Demetriou et al. 2017), and it drives transitions across developmental cycles (Kazi et al., 2019).

AACog integrates Spearman's (1927) eduction of relations (abstraction) and correlates (alignment) underlying g, Piaget's (2001) reflective abstraction and equilibration, and reasoning and consciousness that dominated in post-Piagetian developmental research (Scott et al., 2014). Inference is a fundamental tool in the service of the AACog mechanism. Early in development, Bayesian inference induces (firstorder) relations based on statistical regularities (Tenenbaum et al. 2011). Later, inductive, analogical, and deductive reasoning construct relations at various levels of abstraction based on extrapolation of rules (induction) or deduction from rules about truth and consistency already available (Demetriou et al. 2017).

\subsubsection{Brain Processes Serving Intergative Mental Processes}

2.3.2.1. AACog. The neural analogue of the AACog mechanism would include a sequence of neural networks that would map onto the Alignment, Abstraction, and Cognizance processes of this mechanism. Specifically, there should be (i) networks which would first register information or information patterns (alignment); (ii) then there should be networks where these patterns are projected in order to be associated with relevant information (abstraction); (iii) finally these relations would be made available to another network which makes final choices (an answer, a solution, or a conclusion) which may be a combination, reduction, or variation of representations considered; (iv) a parallel network here may spot for application problems of the networkds above and provide evaluative feedback allowing optimization of choices (cognizance). In the process, any of these networks may call upon networks for support, such as retention or inferential networks networks (e.g., inductive or deductive reasoning). Notably, recent research suggests that focusing on semantic abstract information, such as the notion of apple in Judeo-Christian tradition, recruits a series of networks related to the physical haracteristics of apples (their color, shape, etc.); this interaction between networks indicates a brain search and alignment processes that carries information at a higher level where it is integrated as an abstraction serving a general semantic category (Fairhall, 2020).

This cascade of networks has been recognized several times. Jung and Haier (2007) proposed the parieto-frontal integration theory (P-FIT) as the brain analogue of $\mathrm{g}$. According to P-FIT, information is first registered and processed in regions of the cortex activated by different types of sensory information, such as visual (BA 18, 19) or auditory cortex (BA 22). Therefore, the sensory areas involved in the P-FIT model may be more related to the domain-specific processes represented by the present model, such as categorical, spatial, or quantitative core operations. From there, information is fed 
forward to several regions in the parietal cortex (BA 7, 39, 40), which primarily carry out elaboration, association with past knowledge or action, and abstraction. Therefore, the parietal areas of the P-FIT model seem related to the abstraction and alignment processes in AACog. Then, these regions interact with frontal regions (BA 6, 9, 10, 45-47) in search of alternative solutions to the problem at hand. These appear related to cognizance in the AACog model. Based on recent evidence, Haier (2016) expanded PFIT to include further regions in the medial frontal cortices, such as the anterior cingulate (BA 32) (engaged in response selection by inhibiting alternative responses) (Gruber and von Cramon 2003; Klingberg 1998); the posterior cingulate and subcortical structures, such as the caudate nucleus are also related (Basten et al. 2015).

2.3.2.2 Reasoning. Interestingly, networks associated with inductive and deductive reasoning overlap but are not identical with the networks above. Specifically, simple inference based on Bayesian probabilistic covariation relates to activation in the left cerebellum and adjacent visual cortex; this network seems to integrate relations between movement and visual information (Ide et al. 2013; Zheng and Rajapakse 2006). Bayesian abstraction of functional covariation between events involves, additionally, anterior cingulate and prefrontal cortex, which is always present in relational thought (Ide et al. 2013; Wendelken et al. 2015). Inductive reasoning activates the frontal gyrus (serving integration) and the right insular cortex, serving salience detection and switching between large scale networks. This system activates attention and working memory networks to ensure focusing on and representing the salient representation selected (Menon and Uddin 2010) (see Figure 2).

Deductive reasoning activates several networks serving different tasks at different stages of the inferential process. Specifically, content based propositions activate temporal (BA 21, 22, serving language processing) and frontal regions (BA 44, 8, 9, serving integration). Formal propositions activate occipital regions (BA 18, 19), suggesting construction of visual mental models of the relations implied by the formal propositions, left parietal (BA 40, building associations), and bilateral dorsal frontal (BA 6), left frontal (BA 44, 8, 10), and right frontal (BA 46) regions, serving integration, evaluation, and selection (Goel et al. 2000; Goel and Dolan 2000, 2001). Other studies suggested activation of the right superior parietal lobule (serving associations between concepts), the thalamus (relaying information between systems), and the right anterior cingulate (serving selection of competing responses) (Osherson et al. 1998).

Vendetti and Bunge (2014) proposed that three regions are central in analogical or deductive reasoning. The first, located in the IPL, represents specific rather than general relations and it scales with the number of relations to be considered. This seems related to abstraction as it makes first-order relations available to the second region, rostrolateral prefrontal cortex (rlPFC). This region aligns firstorder relations and abstracts second-order relations, comparing and integrating mental representations over common relations running through them. The third region, dlPFC, provides a supporting role, enabling performance monitoring, interference suppression, response selection and manipulation of items in working memory. The second region does not but the third does scale with difficulty. Thus, this relates to cognizance more than with the other AACog processes. Age-related increases in myelination of the left rlPFC-IPL tract, but not the corresponding right tract, predict reasoning development (Wendelken 2015).

2.3.2.3. Cognizance. There is no consensus about the brain bases of consciousness in general and cognizance in particular. Some scholars maintain that consciousness is associated with specifically dedicated brain structures (Crick and Koch 2005); others claim that it is an emergent dynamic condition reflecting the co-activation of several networks (Baars 1993). It is beyond the concerns of this paper to embark on this discussion. From the point of view of the present concerns, there is strong evidence that some regions and networks are always involved in cognitive awareness. Some aspects of attention, such as selective attention, or focusing on mental representations, as in mental rehearsal, are linked to cognitive awareness because they operate as the gate between unconscious and conscious mental functioning.

However, explicit awareness of mental states always involves the prefrontal cortex. fMRI studies suggest that introspection discriminating correct from incorrect performance involves the prefrontal cortex. Fleming et al. (2010) showed that introspective ability was correlated with grey matter volume 
in the anterior prefrontal cortex. Moreover, interindividual variation in introspective ability was correlated with white-matter microstructure connected with this area of the prefrontal cortex. Experimental inactivation of the prefrontal cortex handicaps metacognitive judgements about performance on a task but spares processing the task. Rounis et al. (2010) used transcranial magnetic stimulation to depress activity in the dorsolateral prefrontal cortex as participants performed a visual discrimination task. They found that participants were able to perform the discrimination task but they were not able to discriminate between correct and incorrect stimulus judgements. In fact, there is theorizing that metacognitive monitoring is one of the major evolved competences of the prefrontal cortex (Carlén 2017; Dehaene et al. 2017) (see Figure 2).

How does this mechanism operate? According to the global workspace model (Baars 1993), a particular object of thought would reach consciousness and become the object of explicit awareness when the corresponding neural population is mobilized by top-down attentional amplification into a self-sustained brain-scale state of coherent activity that involves many neurons distributed throughout the brain. In its modern version, "local specialized cortical processors are linked, at a central level, by a core set of highly interconnected areas containing a high density of large pyramidal neurons with longdistance axons (in parietal and prefrontal areas). At any given moment, this architecture can select a piece of information within one or several processors, amplify it, and broadcast it to all other processors, thus rendering it consciously accessible and available for verbal report." (Mashour et al., 2020). It is as though this particular content seizes the whole brain for some time and is made available to various processes, such as categorization, quantitative estimation, long-term storage, through the long-distance connectivity of these top-down amplified 'workspace neurons' (Sergent and Naccache 2012, p. 102). Evidence suggests that two regions are causally crucial in this process: mPFC and midline parietal cortex (mPC). Inactivating them by anesthesia annihilates awareness (Boly et al. 2013). Below we examine how networks communicate.

Overall, relating, abstracting, and inferring is a stepwise process activating overalapping cortices. This processes evolves over a sequence of steps where information is registered and represented by particular networks, often domain-specific (these may be in visual, acoustic, or cerebellar networks). The abstraction of common patterns is sent to other networks which are sensitive to the patterns (IPL). Then inferential integration of the patterns in terms of inductions or deductions are delivered to other networks (in the PFC) which are responsible to "impose" connections, such as extrapolations of relations induction, or conclusion selection in deduction. These later processes involve awareness and evaluation, where content-laden networks become concurrently available to a cognizance template for inspection, evaluation based on criteria of logical validity or truth.

\subsubsection{Communication between Brain Networks}

So far, we talked about interactions between brain networks related to cognitive processes but we did not specify the neuronal processes underlying these interactions. The second question stated in the introduction was concerned with these neuronal processes. It seems that these processes are expressed in electrodynamic activity taking place at various frequencies. Brain rhythms are periodic oscillations in excitability of groups of neurons as reflected in EEG activity. Rhythms vary from very low (i.e., .05 $\mathrm{Hz}$ frequency) to very high (200-600 Hz). Perceptual and cognitive activity is mainly expressed into delta $(1.5-4 \mathrm{~Hz})$, theta $(4-10 \mathrm{~Hz})$, alpha $(7-13 \mathrm{~Hz})$, beta $(10-30 \mathrm{~Hz})$, and gamma rhythms $(30-80 \mathrm{~Hz})$ (Buzsaki et al. 2013).

It seems that different rhythms are associated with different brain regions, serve different functions, and they are hierarchically organized during cognitive processing. Specifically, alpha and beta oscillations arise in the visual cortex and the thalamocortical system and serve visual perception and related sensory-sensory and sensory-motor coordination (Thut and Miniusi 2009). Beta oscillations also arise in the motor cortex and transfer messages to the motor systems. Gamma oscillations serve multiple purposes. They are associated with local computation but they are also involved in selective and flexible coupling of neighboring and distant regions, such as the hemispheres, to integrate correlated information. Feature binding, perceptual closure, focus of attention, and maintenance of contents in working memory are closely associated with increased beta- and gamma-band oscillations and enhanced synchronization (Irrmischer et al. 2018). Theta oscillations originate in prefrontal- 
orbitofrontal regions and propagate caudally; they also arise in the hippocampus. Slower rhythms, such as theta rhythms, can reset and modulate the power of faster rhythms running local computation in different cortical areas, channeling it to current computational needs. These rhythms constitute the basic components and syntactic rules of brain language (Buzsaki and Brendon 2012; Buzsaki et al. 2013).

For instance, in a sequence of items presented in working memory tasks, such as successive letters or digits, items are encoded by high frequency rhythms, such as gamma oscillations emerging in the hippocampus. Gamma oscillations may be gating only the most excited items to fire, synchronize spikes, and delimit items by creating pauses between them (Lisman and Jensen, 2013). These stand for the neural letters of thought. These letters are combined into "neural words" and neural sentences according to a specific rule, such as their presentation order, which is encoded by lower frequency rhythms, such as theta oscillations, emerging in the prefrontal cortex (Buzsaki and Brendon 2012). Perhaps, the sequence of gamma oscillations in the hippocampus stands for STS; the sequence of theta oscillations in the prefrontal cortex stands for the executive processes, elevating STS into working memory (Jensen and Lisman 2005). Lisman (2005) suggested that working memory capacity equals the number of gamma cycles (standing for individual stimuli) that can go within a theta cycle. Thus, integrated gamma/theta cycles stand for a brain code for storing multiple items in working memory (Segneri et al. 2020).

Others suggested that theta activity is the fundamental integrative mechanism of the brain that coordinates different types of information expressed into other brain rhythms (Sauseng et al. 2010). Maguire et al. (2010) showed that theta power increased over right frontal areas when processing thematic relations between words (e.g., leash-dog); alpha power increased over parietal areas when processing taxonomic relationships (e.g., horse-dog). Along this line Clarke et al. (2018) showed that frontal theta and posterior alpha/beta oscillations play a key role during associative memory formation. Frontal midline theta oscillations were stronger at the beginning of learning and declined with increasing strengthening of stimuli-response associations. At this early phase, posterior alpha and lowbeta oscillations decreased and they increased with strengthening of associations. This pattern suggests that changes in theta oscillations reflect changes in the control needs of the learning task (e.g., better maintenance of cue information, thereby supporting better encoding of the stimulus-response relation); changes in the alpha/beta oscillations reflect changes in the learning attained (i.e., establishment of the stimulus-response relation) and ensuing changes in inhibition needs. In line with these findings, Segneri et al. (2020) recently demonstrated experimentally changes in the amplitutes of theta oscillations force theta-nested gamma oscillations to chane proportionally in the range of 1 to $10 \mathrm{~Hz}$. Noticeably, Reinhart (2017) demonstrated recently that experimental enhancement by means of transcranial alternating current stimulation of the coordination of theta oscillations in two different regions of the prefrontal cortex (medial frontal cortex and lateral prefrontal cortex) improved executive control. Experimental disruption of this coordination weakened executive control. These findings indicate that theta oscillations are brain carriers of executive control. Along the same lines, Jaušovec and Jaušovec (2014), showed that transcranial alternating current stimulation over the left parietal region but not the left frontal region resulted in enhanced working memory performance. These findings indicate that different brain regions use the theta band to exert control on other regions.

In conclusion, the research reviewed above suggests that there are several (overlapping) networks in the brain sub-serving each one of the various systems of the mind; in fact, various brain rhythms are used in the service of the interactions between these networks. Below we will discuss the development of these networks.

\section{3. Development}

\subsection{Generally Accepted Assumptions about Cognitive Development}

Ever since Baldwin (1906) and Piaget (1970), students of intellectual development described a sequence of four stages in cognitive development spanning from birth to adolescence. This classic stage sequence has been extensively investigated and redefined by several scholars, each focusing on a 
Zelazo 2015). Despite differences in emphasis or language, this scholarship converged on several recurrent findings:

Major transitions in the nature of representation or concepts occur around the same age: at 2, when there is a representational spurt culminating in language attainment; at 7, when there is a spurt in mental cohesion and flexibility, culminating in learning to integrate over representational systems to build new complex skills, such as reading and school arithmetic; and 11 years, when there is a spurt in dealing with abstract ideas and using related arbitrary symbol systems, such as algebra (Demetriou et al. submitted). There may be variations across domains, but these changes are fairly independent of tasks or methods of analysis. To integrate over theories and empirical findings, we suggested a fourcycle developmental sequence, with two phases in each. New representations emerge early in each cycle and their integration dominates later. The four cycles differ in their dominant unit of representation. They are as follows: (1) interaction episodes and modality-specific memories; (2) language (words) and mental images; (3) mental blueprints and action scripts defined by rules, sometimes symbolized by artificial symbol systems such as numerals; (4) canonical principles prescribing acceptable possibilities, sometimes symbolized by highly idiosyncratic symbol systems, such as notation in different sciences.

The emergence and consolidation of a new unit of representation in each cycle sparks the emergence of new representational systems which mark transition across cycles: (i) speaking at 2; (ii) reading, writing, and arithmetic at 6; (iii) abstract idiosyncratic symbolic systems, such as advanced notation in mathematics, science, or arts, at 11 years. New symbol systems open new possibilities for the representation of increasingly complex states of the world. Thus, representations in successive cycles differ in resolution, predication, symbolizability, connectivity, and cognizability. This is basically the reason why cognitive constructs become increasingly abstract, complex, and logical with development. The term "cycle" over "stage" or "level" emphasizes that development is a continuous process systematically transforming representations and mental processes rather than a succession of steady states. The ages above are modal ages capturing the development of the majority of individuals. There may be both intra- and inter-individual differences in the rate of development across domains or processes, indicating continuity rather than discontinuity in development (Siegler 2016).

These cycles are outlined below with an emphasis on executive control, cognizance, and reasoning. It is emphasizied that developmental priorities change across cycles. Specifically, different mental processes dominate in successive developmental cycles depending upon the functional and adaptive needs of each phase. Overall, g gradually shifts from executive to inferential and selfawareness processes. The cognitive profile of different age phases constrains what can be learned and what problems can be solved (Demetriou \& Spanoudis, 2018; Demetrtiou et al., 2017, 2018). The reader may consult other sources for a discussion of the relations between this sequence and other theories (Demetriou and Spanoudis 2018; Demetriou et al. 2017 2018).

\subsection{Developmental Cycles in Cognitive Development}

Episodic representations are blueprints of actions and experiences preserving their spatial and time properties. Interaction control is the major developmental priority of this cycle: infants must learn to interact with objects and persons through different modalities, capitalizing on the affordances of the environment and their own bodily possibilities and skills (Thelen \& Spencer, 1998). Interaction with the environment generates episodic representations. There is no executive control until 4-5 months of age; directed attention and inhibition appear systematically in the 6-9 months window (Holmboe et al. 2017). By this age, attention-based actions may be initiated by interesting stimuli or action sequences may be restoted. Along with the second year of life, recurring event sequences are aligned into episodic representations yielding mental blueprints of action (e.g., follow, manipulate, recover and coordinate objects and actions) and inference (Carey 2009). Awareness is still minute and transient. However, by 15-18 months, infants show awareness of actions performed; they seem to demonstrate an executive sequence where past actions are intertwined with perceptions and current actions, indicating awareness of the contents of activity (Dehaene et al. 2017). Reasoning as such does not exist in this cycle (Xu, 2020). 
Episodic reasoning involves reciting episodic representations or actions in sequences predating reasoning schemes such as conjunction (e.g., "I put this, and this, and this, all of them"), disjunction (e.g., this one, not this one), or implication ("first this then this) (Demetriou and Spanoudis 2018). However,

Realistic representations. At the end of the second year, episodic representations are projected into realistic mental representations. These are blueprints of episodic representations distilled from initial spatial and time properties, associated with symbols, primarily mental images and, increasingly, with words. Control of attention is the major developmental priority of this cycle. Holding representations active and in focus so that information in the senses is encoded, processed, chosen or ignored, according to its relevance to the currently focused goal so that an action sequence may implement a mental plan. By the age of 3, children can subject their behavior to the control of a represented goal and implement the actions required, as in go/no go tasks (Diamond 2013; Zelazo 2015). In the second phase of this cycle children demonstrate representatonal awareness in several domains. Thus, attention control and representational awareness are major markers of $\mathrm{g}$ in the 4-7 years period (Demetriou et al., 2017). Children now understand that perception is a source of knowledge (Spanoudis et al., 2015). In ToM tasks children understand that different individuals may have different representations, depending on what they perceive (Wellman 2014). By the end of the cycle, representational awareness is a crucial factor for learning to read and understand arithmetic (Peters and De Smedt 2018). Reasoning emerges in this cycle but is secondary to attention control and representational awareness. Representations may be aligned into sequences yielding conceptual inductions (e.g., he barks, he is a dog) and "plausible deductions (e.g., "it's cloudy"; "it will rain"). However, there is no explicit awareness of the logical links between representations.

Rule-based thought. At 6-8 years, with attentional control established, links between representations begin to emerge; language predication contributes to establishing these links. This complexity presents a new developmental challenge: identify relations between representations and organize them so that they can be called upon efficiently in sake of understanding and interaction. The major task of this cycle is to master the process dealing with relations between representations. Thus, inferential control is the major developmental priority in this cycle. In this cycle children begin to use rules in solving inductive and deductive reasoning. In inductive reasoning, which is a major marker of $\mathrm{g}$ in this age period (Demetriou et al., 2017), children make the relational shift, looking for relations between relations (Gentner 2005). In deductive reasoning, they reach bi-conditional reasoning where modus ponens ( $p$ and $\mathrm{q} ; \mathrm{p} ; \mathrm{q}$ ) and modus tollens ( $\mathrm{p}$ and $\mathrm{q}$; not $\mathrm{q}$; not $\mathrm{p}$ ) are integrated into a common scheme (Barrouillet and Lecas 1999). However, they still fail logical fallacies. These changes come with cognitive awareness in several domains and flexibility in shifting between them. Children become explicitly aware of inference as a tool for creating knowledge and linking representations. (Kazi et al., 2019; Spanoudis et al., 2019). They differentiate between easy and difficult memorization tasks, suggesting awareness of the relation between complexity of representations and learning (Chevalier and Blaye 2016).

The principle-based thought. Truth control is the developmental priority of principle-based though. Thus, adolescents begin to command conditional reasoning, resisting logical fallacies, which is a major marker of $\mathrm{g}$ in this cycle (e.g., $\mathrm{p}$ and q; not p; unknown if q) (Christoforides et al. 2016; Demetriou et al. 2017). The principle-based mind adopts a suppositional stance, viewing realities from multiple perspectives. They also gradually grasp the principles connecting inferential rules (Makris et al. 2017). Early in adolescence, at 11-13 years, adolescents begin to form accurate maps of mental functions and their own strengths and weaknesses. As a result, they evaluate their own performance on cognitive tasks with increasing accuracy. Control in this cycle is based on a cohesion and validation program allowing to co-activate conceptual spaces, evaluate them against each other, and form long-term life plans (Moshman 2011).

\subsection{Changes in the Integrative Processes of the Mind}

Integrative processes emerge with development. In Fodor's (1975) terms, a Language of Thought (LOT) is not innate; it is gradual output of the reorganization of mental processes in intellectual development. Its basic proto-representational units are generated by the core operators within each of the domain-specific systems and they then evolve into the basic representational units of each cycle 
(episodic, realistic mental, rule-based, and principle-based representations). The control priority of each cycle defines the syntactic rules of LoT integrating representations in each cycle. In infancy, episodic .representations are integrated in the flow of episodic events. Thus, the attention-based transient and fragmented awareness of action episodes generates proto-reasoning experiences, such as recursions (e.g., "and ... and ... and ...") or choices between events or objects (or ... or). However, infants cannot be credited with reasoning because these associations are not represented as such (Xu, 2019). The attention control program of the representational cycle adds a representational dimension to the interaction control program of the episodic infant, enabling preschoolers to develop parallel mentally based action plans in addition to stimulus initiated action plans. Explicit awareness of representations and their streaming according to a goal in realistic representational thought yields explicit inductions and pragmatic deals. However, inference is still embedded in realistic sequences rather than deduced. The inferential control program in rule-based representations adds an inferential dimension to executive control allowing to coordinate mental spaces in addition to representational-action inhibition processes. Inferential control in rule-based thought generates awareness of rules, allowing systematic premeditated reasoning which culminates in the deductive reasoning of the adolescent (Barrouillet and Lecas 1999; Reverberi et al. 2012). The truth control program in adolescence adds an epistemological dimension to executive control, allowing control based on a long-term time-scale integrating the past, the present, and the future into a cohesive plan.

\subsection{The role of cognizance in the development of integrative processes}

Cognizance mediates between executive control and reasoning from infancy to adulthood. This mediation is cycle-specific, exerted through the processes underlying the management of representation in each cycle: perception-based aspects of representation in the representational cycle, rule-based inferential processes in the rule-based cycle, and abstract semantic processes in the principlebased cycle. This implies that cognizance is a regulatory process registering representations and participating in their metarepresentation into new more abstract and thus flexible forms. In terms of the AACog mechanism, alignmenents and abstractions come increasingly under the influence of cognizance so that they shift from the local environment of core processes to a kind of mental universalism where rules and principles abstracted in the past may be systematically invoked to evaluate or fill in lags of current representational ensembles.

In line with this assumption, recent longitudinal evidence showed that cognizance drives developmental momentum of executive and reasoning processes from 4 to 11 years (Kazi et al., 2019). Bottom-up mediation is stronger early in development; in the period from about 4 to 7 years, executive processes, attention control, and working memory in particular, generate awareness of mental processes that is used in managing reasoning. Top-down mediation appears from late childhood onwards; at 8-10 years, awareness emerging from reasoning as such is transferred top-down to executive control, enhancing its scope and flexibility (Demetriou et al. 2018; Spanoudis et al. 2015).

\subsection{Developmental Changes in the Brain}

The third question asked how brain changes relate with cognitive developmental changes. It is well established that brain undergoes both structural and functional changes in development, in several dimensions, including its sheer volume, condition of the neurons, inter-linking within and between regions, and biochemical functioning, including neurotramitters and electrical activity. These changes reflect a dynamic interplay of simultaneously occurring progressive events, such as increases in neuronal matter and synapses, and regressive events, such as cell death and synaptic pruning (Millán et al., 2018). A crucial prediction of the model of the mental architecture and development above is that these changes in the brain must be connected to both, the various systems specified by the architecture and the developmental sequences specified by the developmental dimension of the model. 
The sequence of cognitive changes above suggest that domain-specific processes mature first; attention control processes start to mature in the second year of life but they culminate as attention control and representational awareness processes from 3 to about 6 years; inductive reasoning processes take off in middle childhood at about 7-8 years and they culminate as deductive logical reasoning in adolescence at 13-15 years. We therefore, expect a pattern of changes in the brain networks associated with each of these processes at the corresponding age windows. That is, one would expect that sensorymotor cortices underlying domain-specific core processes would mature first; then these would interlink with parietal associative cortices and attention control and mentalizing networks; then both sensory and parietal cortices would interlink with frontal cortices underlying integrative inductive and analogical reasoning networks; finally, specific cortices related to self-awareness and self-evaluation may emerge. Below we will first review research related to developmental changes in brain structures and networks. We will then focus on changes in brain functioning.

Total brain size increases drastically from birth to middle childhood, reaching about $90 \%$ of adult size by age 6 years. Synaptic density (i.e., the number of synapses/mm3) changes exponentially up to the first year: it is about $.5 \times 108$ at the fifth gestational month and it increases to $2.5 \times 108$ at birth to 5.8 x 108 at the end of the first year; it then slows down to $3.5 \times 108$ at 10 and $3 \times 108$ at 70 years). In line with our predictions, synaptogenesis and synaptic pruning underlying brain networking differs across brain regions. Overall, slow wave activity, an index of increases in cortical plasticity, starts early in posterior areas and spreads rostrally to the frontal areas through the first two decades of life (Kurth et al. 2010). The various maturation patterns are consistent with the representational and procedural profile of successive cognitive developmental cycles. Specifically, spontaneous patterned brain activity starts before birth. This activity plays an important role in the formation of the brain networks needed for the activation of perception at birth (Sporns 2016). Moreno-Juan et al. (2017) showed that prenatal thalamic spontaneous calcium waves are key regulators of sensory cortical area size and that prenatal alteration in inter-thalamic sensory nuclei communication may trigger size adaptation in cortical fields. Hence, acoustic, visual, and motor networks develop fast in infancy, enabling the construction of episodic mental units (Franson et al. 2011).

It is notable that these changes occur in a context where the scaffold of developing brain networking, as indicated by resting state networks, is in place before birth, with rapid neural growth in the last three gestational months (Hoff et al. 2013). This scaffold includes bilateral primary motor, primary visual and extra-striate visual, parietal-frontal and frontal (executive control networks) insulartemporal/anterior cingulate cortices (ACC; salience and shifting networks). Also, the basics of the default-mode network (DMN), including the posterior cingulate cortex (PCC), the medial prefrontal cortex (mPFC), the medial temporal lobes (MTL), and the angular gyrus (AG), is in place since very early but develops throughout childhood. Superkar et al. (2010) found that the PCC component is present from the second week of life. By the age of 7-9 years, functional connections between PCC, mPFC, bilateral MTL and bilateral AG attain adult levels. Up to this age, there is an excess of grey matter in children which is pruned in favour white matter in adulthood, indicating stronger structural connections.

Attentional, associative, executive, and reasoning networks follow a long developmental process extending to early adulthood. Initially, the attentional orienting network is well active by the age of 67 months, allowing attention shifting and focusing as a result of changing stimulation in the environment. This network develops throughout the first and the second years of life, allowing episodic executive control needed to look for and recover hidden objects at the age of 18-20 months (Thompson et al. 2000). These connections are strengthened throughout childhood. Also, in the period between 3 and 6 years there is a drastic change (60-80\% percent increase in connecting fibers) in the frontal circuits of the corpus callosum, which sustain vigilance and regulate action planning. This goes well with the finding that in this phase, at 3-4 years, the inhibition network is effectively functioning, rendering the attention control program of the representational cycle possible. Probably these changes are also related to a spurt in visual working memory and overall representational activity, including imitation and attraction to imaginary stories that preschoolers demonstrate. Later, at 6-7 years, there is dramatic change in the callosal isthmus, which supports intraparietal associative and language functions. Also, 
in the period from 7-11 years the anterior cingulate gets differentiated from the orienting network and connects to the executive and the associative IP networks (Rothbart and Posner 2015), perhaps related to the conceptual fluency program attained in this period.

Attentional networks continue to develop throughout childhood. According to de Bie et al. (2012), a cingulo-opercular attentional network appeared fragmented into the two components even at age 8 years: the first component starts from the inferior frontal gyrus (IFG) and extends into the middle frontal gyrus bilaterally; the other comprises the cingulate gyrus extending into the medial superior frontal gyrus, bilateral insula/frontal operculum of the IFG, and bilaterally in the superior frontal gyrus, precuneus, and thalamus and cerebellum. These networks integrate into a single network in adults, which also involves the PCC/precuneus in the ventral frontoparietal network. This network relates with goal directed behavior, salience processing, and cognitive flexibility and it may relate to improvements in executive control attained in adolescence. Similar trends were observed in the development of working memory. Scherf et al. (2006) showed that 10-13-year children only minimally relied on core working memory regions when performing a working memory task; instead, they relied primarily on ventromedial regions (caudate nucleus and anterior insula). The working memory network emerged in adolescence but it was diffused (dlPFC, anterior cingulate, posterior parietal, anterior insula), including premotor response preparation and execution circuitry. In adults ( $>18$ years) this network narrowed down into the specialized working memory circuitry which interlinked with performance enhancing regions, including left-lateralized dlPFC, ventrolateral prefrontal cortex, and supramarginal gyrus

Several studies investigated the development of the mentalizing network (TPJ, superior temporal gyrus (STS)/middle temporal gyrus (MTG), PC and mPFC) associated with ToM and false belief understanding. Specifically, Wiesmann et al. (2017) explored the white tracts structural connections in this network comparing 3-4-year old children who succeeded with their agemates who failed on ToM tasks. They found that age related changes in TemporoParietalJunction, MedialTemporalGyrus, PariatalCortex and MedialPreFrontalCortex and also age-independent streamlines between temporoparietal and inferior frontal regions were related to grasping ToM. Interestingly, controlling for associations of these networks with executive function and language performance showed that some parts of the networks are specific to ToM (the right TPJ, left MTG, right MPFC and right PC) and they are independent of executive function and language. Notably, Sabbagh et al. (2009), exploring functional rather than structural connections as expressed in the alpha band sequence, they found that basically the same network (dorsal medial prefrontal cortex (dmPFC), right temporoparietal junction (rTPJ), cuneous, inferior temporal lobe) is associated with ToM in 4-year-old children. They theorized that dmPFC performs the computations associated with complex metarepresentational reasoning; the rTPJ performs the highly specific task of reasoning about representational mental states; the cuneus is engaged when reasoning about self-knowledge and it may also provide support via mental imagery when recalling the foregoing events; the development of inferior temporal regions may contribute to the development of the other regions.

Some parts of this network [lateral prefrontal cortex (IPFC) and medial posterior parietal cortex (mPPC)] are also associated with reasoning as such. Wendelken et al. (2015) found systematic changes in brain networks associated with first- and second-order reasoning. Specifically, in the 7-10 years cycle, the IPL-rlPFC network is activated but it undifferentiably processes both first- and second-order relations. This differentiation occurs in several phases. In the 6-8 years phase, reasoning development goes with weakening of connectivity between the dlPFC and the ventrolateral prefrontal cortex (vlPFC). In line with our findings about the recycling of relations between speed and working memory, on the one hand, and reasoning, on the other hand, in the 6-8 years phase, changes in speed mediated changes in reasoning ability, suggesting that entering the cycle of rule-based reasoning is associated with differentiation between dIPFC and vlPFC, causing faster processing. In the next phase, in 9-11 years phase, the rlPFC dominated and it was coupled with the right rlPFC. Notably, in this age phase, changes in working memory mediated changes in reasoning, suggesting that consolidation of rule basedreasoning is associated with the consolidation of the relevant brain network which is expressed in working memory expansion. In the 11-14 years phase, left and right dlPFC as well as dorsomedial PFC were more strongly engaged in processing second-order rather than first-order relations. That is, in this 
phase, the second pole takes its primary function in processing second-order relations. In the 15-18 years phase, left rlPFC and bilateral IPL were engaged in processing second-order relations as contrasted to first order relations. It seems that cortical reorganization within the IPL leads to greater efficiency in processing first-order relations, thereby reducing relational processing demands within rlPFC. Notably, Mackey, Miller Singley, and Bunge (2013) found that training in reasoning strengthened the connections between the rIPFC and the IPC but also between the IPL and the striatum. One may see that changes in relations between brain networks and reasoning from 7-15 years coincides with the cycles of rule-based and principle-based though.

Changes in other networks apparently related to reasoning develop from middle childhood to adolescence. Wendelken et al. (2017) showed longitudinally that increases in structural connectivity between the rlPFC and IPL are fastest in middle childhood, with maximal rate of increase at age 7 years; these structural changes preceded functional changes between these regions, which started in late childhood and peaked at age 13 years. In fact, earlier increases in structural connectivity predicted later increases in functional connectivity. Also, some of the changes in structural and functional connectivity were related to changes in reasoning: increases in structural frontoparietal connectivity were strongly related with reasoning development in the period from 6 to 12 years. Therefore, improvements in structural connectivity in this network was related to changes in reasoning ability during the age period of rule construction; improvements in functional connectivity concurred in adolescence, when principle-based reasoning develops. However, development goes beyond adolescence. Marek et al. (2015) showed that the networks linking the ventral and medial prefrontal cortex to limbic and temporal regions continue to change into young adulthood. It seems that the top-down control network is fully established only in late adolescence and early adulthood.

Networks are more diffused early in development. Güler and Thomas (2013) used event-related fMRI to compare brain activation at 8-9 and 12-13 years when learning to memorize and recall unrelated pictorial pairs (e.g., hat-giraffe, balloon-window, etc.). There were some clear differences in both encoding and recall. Younger children recruited wider regions in the right dorsolateral prefrontal and right temporal cortex compared to adolescents during successful encoding of the pairs. However, during successful recall, adolescents recruited wider regions in the left ventrolateral prefrontal and left inferior parietal cortex compared to children. These results suggest that prefrontal cortex is involved during both encoding and recall but the exact activation changes in development. Younger children need more regions bilaterally to sustain encoding. With age, building relations becomes more efficient and this is reflected in activating special dedicated regions; thus, encoding becomes more focalized because it is more efficient.

\subsubsection{Cycles in the Functional Aspects of Brain Development}

The patterns discussed above would imply corresponding patterns in the development of brain rhythms. Specifically, alpha and beta rhythms, emerging from early maturing sensory and motor cortices should mature before rhythms, such as gamma and theta, emerging from cortices maturing later. Gamma rhythms, which are important in the integration of widely distributed brain modules, including thalamocortical and corticothalamic networks involved in control and inhibition, should accelerate in the 2-6 years phase, when representations spurt and get aligned. Finally, delta and theta bands should peak in late childhood and adolescence, when top-down control culminates. The evidence below appears consistent with this expected pattern.

Notably, brain changes tractable in EEG recordings appear to match the intellectual cycles outlined here. Thatcher $(1992,1994)$ found that electroencephalographic coherency, which reflects changes in connections within and between regions, develops in growth spurts that are nearly identical to the time frame of the developmental cycles described above: four cycles extending from early childhood onwards occur at approximately 1.5-5 years, 5-10 years, and 10-14 years of age, with growth spurts occurring at the ages of 6, 10, and 14 years. Hudspeth and Pribram (1992) reported changes in relative power indices across the brain occurring in stages almost perfectly matching those presented by Thatcher and the cycles proposed here (1-6, 6-10.5, 10.5-13, and 13-17 years). Epstein (1986) showed that 
spurts in total EEG energy in the alpha band (8-13 Hz) coincide with the first phase of the cycles presented here: They occur at the age of 2-4, 6-8, 10-12 and 14-15 years.

At a finer level, changes in different rhythms seem to align with the predictions above. Theta activity is present from infancy. Bosseler et al. (2013) showed increases in theta rhythms at the age of 12 months as a response to native syllables, which was not observed in 6-month-old infants or adults. This was interpreted to imply that by this age children recognize their native language and they have their attentional network engaged to process a meaningful sound pattern. Overall, however, there seems to be a shift from low frequency power $(<6 \mathrm{~Hz})$ to higher frequency power $(>6 \mathrm{~Hz})$ from infancy to early childhood. Specifically, it seems that there is a peak of alpha power at $6-10 \mathrm{~Hz}$ at 10 months of age capturing central sensory-motor activity. This band reaches a limit at $9 \mathrm{~Hz}$ at the age of four years (Marshall et al. 2002).

Gamma rhythms emerge in early preschool age and peak in middle childhood. Benasich et al. (2008) found that there is a large increase in gamma power from 16 months to 36 months of age. This increase was systematically correlated with various aspects of language learning, such as auditory comprehension and speech production, and cognitive abilities such as attention shifting and inhibition. Moreover, changes in gamma power over this period clearly differentiated between children with a family history of language impairment from children free of such history: gamma power grew stronger with age in this latter group of children. Notably, these researchers showed in a longitudinal follow up of their sample, that gamma power in the 2-3 years period strongly predicted language (syntax comprehension) and cognitive development at 4-5 years of age (Gou et al. 2011).

Changes in low band control rhythms appear to vary with developmental phase, as expected. Specifically, Orekhova et al. (2006), showed changes in theta and mu rhythms from infancy (6-12 months) to preschool years ( 3 to 6 years) in both power and reaction to different types of stimuli. Overall, theta band changed from $3.6-5.6 \mathrm{~Hz}$ in infants to $4-8 \mathrm{~Hz}$ in children and mu band changed from 6.4-8.4 Hz in infants to 8.4-10.4 Hz in children. Also, there were some notable differences and similarities in activation patterns between infants and children. First, infants showed a more widespread theta increase in response to stimulation than preschool children, suggesting reduction and narrowing of task-related cortical activation in development. Second, exploration of unfamiliar objects (attractive toys) caused a predominantly frontal increase of theta power in both infants and children, suggesting control of self-generated goal-related actions. Third, the theta response to social stimulation (social talk in infants and story-telling in children) was strikingly different: theta activity increased predominantly in frontal areas in infants and over parieto-occipital areas in children; this possibly implies stronger control needs in infant to cope with the social interaction and more meaning making and story-related (associative) mental activity in children.

Later in the transition from representational to rule-based though, from 5 to 12 years of age, the relative power of low (delta and theta) vis-a-vis high (alpha and beta) rhythms changed. Somsen et al. (1997) found that power in the delta and theta gradually decreased while power in the alpha and beta changed very little over this age period, implying systematic shift of functioning from sensory-motorbased to control-based rhythms in the transition from representational to rule-based thought. Recently, Rodríguez-Martínez et al. (2015) showed that decreases in theta and beta bands from 6 to 26 years are correlated and they may be related to neuronal pruning. Along this line, Hwang et al. $(2012,2016)$ explored coupling of beta and alpha bands in the dIPFC and FEF in adolescents and adults asked to inhibit automatic saccade eye movements. They found no age difference in the beta band activity associated with dIPFC functioning, suggesting that this region attains adult levels by adolescence. However, adolescents were weaker in functional inhibition. This was associated with decreased alphaband power in the FEF and weaker cross-frequency coupling between dIPFC and FEF, implying limitations in the ability to communicate task-control signals from the dIPFC general control center to FEF which specializes in the control of eye movements. This pattern might imply that gradually beta oscillations come under the control of low band oscillations but this remains to be directly demonstrated.

\section{$4 \quad$ Individual Differences}


The fourth question asked if there are systematic individual differences in brain architecture and functioning that may be connected to individual differences in intellectual functioning and development. Total brain volume is moderately ( .30) correlated with intelligence (Gignac and Bates 2017). However, the relation is more complicated than this correlation would suggest. For instance, with increasing intelligence or expertice, the brain areas activated to serve a mental task become more refined (Haier, 2016). Overall, the various components or material aspects of the brain are differentially related to cognitive functioning.

Also, individual differences in intelligence are related to brain functioning as such. EEG patterns are highly heritable and so idiosyncratic that they may be used for the fingerprinting of individuals (Buzsaki et al. 2013). There is evidence that highly intelligent (>120 IQ) and less intelligent individuals (<90 IQ) differ in several aspects of brain oscillations. First, more intelligent individuals demonstrate more powerful (higher amplitude) EEG patterns. Second, decreased coherence correlated positively with intelligence. This implies increased spatial differentiation and increased complexity of the brain allowing faster and efficient processing. Third, phase delays, which reflect speed of response of a brain region, are not uniform across the brain. More intelligence individuals show very small phase delays in frontal areas and longer delays in posterior areas. This pattern indicates an efficient frontal command system which swiftly allocates processing to a local integration system which takes on domain specific processing, consuming time and resources (Thatcher et al. 2005). Fourth, there is an optimal balance between phase shift duration and phase lock duration associated with intelligence. Overall, in high intelligence individuals, phase shift (associated with inhibitory activity) is longer and phase lock (associated with excitatory activity) is shorter than in less intelligent individuals. Intelligent individuals give enough time to spot and activate neural assemblies relevant to a task but they are flexible enough to unlock from activated assemblies and shift to new ones. Very fast phase shifting may fail to activate resources and slow shifting may increase noise (Thatcher et al. 2016).

Also, there are extensive changes in development. In the fashion that less intelligent adults activate brain networks more grossly than more intelligence adults, networks are more diffused early in development. Güler and Thomas (2013) used event-related fMRI to compare brain activation at 8-9 and 12-13 years when learning to memorize and recall unrelated pictorial pairs (e.g., hat-giraffe, balloonwindow, etc.). There were some clear differences in both encoding and recall. Younger children recruited wider regions in the right dorsolateral prefrontal and right temporal cortex compared to adolescents during successful encoding of the pairs. However, during successful recall, adolescents recruited wider regions in the left ventrolateral prefrontal and left inferior parietal cortex compared to children. These results suggest that prefrontal cortex is involved during both encoding and recall but the exact activation changes in development. Younger children need more regions bilaterally to sustain encoding. With age, building relations becomes more efficient and this is reflected in activating special dedicated regions; thus, encoding becomes more focalized because it is more efficient.

\section{Conclusions}

We summarized a theory about the architecture and development of the mind and reviewed research on the architecture and development of the brain. This integration is comprehensive and new in that it bridges cognitive developmental theory and research with current research on brain organization, functioning and development. Despite the complexity of the theory, the main idea is simple: brain is an integrated modular system where modules specialize to represent specific aspects of the environment and abstract patterns for each aspect; in humans, there are power abstractionreduction processes generalizing over abstractions, offering an increasinbgly highly integrated representation of the environment. These properties are reflected in the organization and development of the developing mind, substantiating psychometric and developmental hierarchical models of cognition and development. Admittedly, this proposed model is vulnerable to criticisms of being weakly grounded on empirical evidence directly related to many of the proposals advanced. It is generally recongized that a general weakness of current brain research is that it amassed large volumes of empirical evidence about brain organization, functioning, and change, but this evidence is not 
integrated into a clear and explicit theoretical framework. This is proposed here, recognizing that the integration may be criticized for weak point-by-point relations between mind entities and brain entities. Below we summarize the main trends and point to the major weaknesses of the endeavor.

\subsection{Mental and Brain Architecture}

The main ternds are as follows. First, the human mind involves (i) several specialized domains of thought and central (ii) control, (iii) integrative, and (iv) cognizance processes. These mental functions and their interactions are served by several overlapping brain networks. Specifically, (i) several networks rooted in sensory cortices but also extending into various other regions serve the core processes comprising each of the psychological domains. For instance, the anterior temporal lobe may be the semantic hub for the categorical system (Patterson et al. 2007); the angular gyrus in the parietal region may be the hub for the numerical system (Dehaene 2011); the claustrum may serve the visuospatial system (Crick and Koch 2005). (ii) Other networks serve control and retention by focusing on and protracting information in time, so that it can be related to preceding or following information. These are rooted in the hippocampus, the reticular formation, and several frontal areas. (iii) Other networks, mainly rooted in temporal, parietal, and prefrontal cortices, take inputs from the networks mentioned above, align them, and abstract their common elements. These networks are associated with reasoning. (iv) Finally, other networks, rooted in frontal and medial cortices, monitor, orient, select, and regulate the networks above to optimize goal-related abstractions. Information entering this last type of networks is available to awareness and metarepresentation.

Two points need to be emphasized here. First, mental functions are served by overlapping hierarchically organized brain networks rather than regions. Although each network may have a preference for some regions rather than others as their primary entry basis, mental functions are associated with networks rather than regions. Regions are used or reused by multiple networks at multiple spatial scales and they participate in multiple overlapping neural coalitions (Anderson 2014). For instance, entry regions feed associative and abstraction regions with sensory-based content enabling rule- and principle-induction; in turn, these later regions, when activated, call upon the earlier regions to offer exemplary content to substantiate the rules or principles in use, grounding them in the real world (Cao et al. 2019). In fact, there is recent evidence that the same nodes change their network membership according to current cognitive state, suggesting that networks change fluntly according to current cognitive needs (Salehi, Karbasi, Barron, Scheinost, \& Constable, 2020). Evidently, neural reuse is an important functional aspect of the brain as such. The very process of letting different networks to overlap underlies brain's capacity to form specific representations of the environment because one component of a network may stand for another component, yielding representational sequences varying in scope and depth.

In line with this assumption, new methods of analysis suggest that the brain is hierarchically organized from networks in the sensory cortices representing perceptually-based concrete information to networks "deeper" in the brain that are increasingly remote from concrete information, representing abstractions. Network-depth analysis suggests that progressive functional abstraction over network depth may be a fundamental feature of the brain (Taylor et al., 2015). Overall, there seem to be several layers of abstraction in networks. Sensory cortices in the bottom are closer to real inputs and they are more modular. Middle regions, including the hippocampus and MTL, are related to memory and have intermediate distance from sensory inputs. Deep regions, least connected to real inputs, include bilateral inferior temporooccipital, bilateral frontal poles, left cingulate anterior, and left inferior frontal pars triangularis, which may support more abstract cognitive functions, such as reasoning. These networks are activated by practically all sensory or core-specific networks (Yue et al. 2017). Noticeably, symbol creation increases as a function of network depth (and thus abstraction), suggesting that increasing abstraction causes increasing metarepresentation.

It is reminded that the early functioning of domain-specific core processes together with their association with specific brain networks has been considered a strong sign that they are innate (Mahon and Caramazza 2011; Carey 2009). The model outlined above suggests that core processes are never free of general processes. Notably, recent research in deep neural networks suggests that the very 
operation of domain-specific networks may be the result of the operation of a general mechanism in the brain that recognizes and encodes recurrent patterns of information in the environment, related to each of the core processes (Testolin et al., 2020). On top, the emergence of mental operations from core processes indicates a general mechanism that supercedes specific core processes embedding them into more general networks. In evolutionary terms, one might suggest that different modules, such as olfaction, hearing, and vision, evolved as information-specific encoders with inherent generalization functions in sake of their optimal adaptive functioning over very extended time scales (Striedter, 2005). Along this line, recent evidence suggests that Broca's area, traditionally associated with language, involves, additionally to a language network, compomnents associated with domain-general multipledemand network (Fedorenko and Blank, 2020). Thus, modules are generalizers by construction. Additing the one over the other in evolution necessitated a supergeneralizer that would connect and integrate information across these information-specific encoders. These are the higher-level networks noted above.

It is important to understand the role of consciousness in this processes. There is recent evidence showing that consciousness is sensitive even to processes going on below the threshold of awareness. This system generates a kind of blind insight reflected in the operation of attention and inhibition mechanisms. Blight insight emerges from interactions among predictive processes which yield topdown predictions about the causes of information coming to the senses (e.g., a particular color or face is expected) and bottom-up projections signaling mismatches between expected and observed stimuli (e.g., this was not the color or the face I expected to see). These interactions are registered and metarepresented allowing systematic self-directed improvement of action in situations where attention is needed (Scott et al. 2014). Tran and Pashler (2017) showed that implicit learning of skills involving fast action, such as tennis learning, involves awareness. Only individuals who explicitly verbalized the rules linking cues to locations learned such predictive rules. Therefore, there may be a cognizance system in the brain (Anderson and Fincham 2014) which "creates, modifies, and rehearses declarative representations of cognitive procedures." (p. 25), intervening at any of the steps involved in problem solving, thereby creating and inter-relating new representations. Noticeaby, there is evidence that metacognitive confidence evaluations of the accuracy of perceptual discriminations are associated with enhanced functional connectivity as reflected in beta synchrony between motor areas and prefrontal regions (Wokke, Achoui, \& Cleermans, 2020). This suggests that metacognive awareness integrates sensory evidence and information about ongoing interactions with the world.

\subsection{Mental and Neuronal Processes}

What are the neuronal processes implementing mental processes and mechanisms? It seems that different mental processes associate with different brain rhythms. Entry level processes associated with core operations and specific attention and retention processes are associated with high frequency rhythms (i.e., beta and gamma). Integrative processes, such as reasoning and awareness, are associated with lower frequency rhythms (i.e., delta and theta). These differences also reflect differences in the regions involved (i.e., caudal and central vs. rostral and ventral).

The brain analogue of AACog lies in the syntactic processes of the brain. Mental comparisons between representations are expressed in oscillatory co-activations between the networks representing the mental entities involved. Ideally, complete rhythm coupling would signify representational alignment. The brain equivalent of abstraction may be the lock of the rhythms coupled above through a rhythm of a different band, such as when several gamma oscillations are bridged by a theta oscillation and the creation of specific markers associated with each abstraction level, standing for commonalities between representations. Cognizance may emerge when this new theta oscillation is made available into a broader theta- or delta-base network, thereby functioning as an autonomous token of a new mental object. Focusing on this new object itself may bring it into the focus of awareness. Ensuing brain activations may be the equivalent of this awareness. Rostrolateral prefrontal cortex is the region primarily serving these needs (Dumontheil 2014). It is reminded that direct alteration of theta band coordination between frontal regions improves executive control, meaning that theta-based brain 
activity can directly alter, top-down, brain and related mental activity based on bands of higher frequencies directly related to perception or action (Jaušovec and Jaušovec 2014; Reinhart 2017).

\subsection{Mental and Brain Development}

How are developmental changes in the brain reflected in developmental changes of cognitive processes? Research suggests that sensory cortices mature early in life, allowing the development of episodic cognition. A control scaffold extends for years in the development of the brain allowing the rescaling of control at increasingly more inclusive levels. Information maintenance cortices, such as the hippocampus, mature later in infancy and the preschool years, allowing representational encoding of episodic structures. Associative cortices continue to mature well into late childhood and to establish connections with frontal cortices, allowing rule abstraction.

We focus on the development of the skeletal control network which seems to scaffold development in various functions. The dominant networks are located in the sensory and the motor cortices, the reticular and the parietal, the prefrontal and the frontal cortices, respectively. The crucial aspect of their expansion lies in the addition of extra connections to the parietal and the frontal hubs, integrating and extending the brain network of the previous cycle. We may assume that at each next cycle some further links are added to the left frontoparietal network (lFPN) involving the dIPFC, the IPL, and the rlPFC specified by Vendetti and Bunge (2014). These links encode further relations in a hierarchical executive or reasoning sequence. Additionally, the cross-coupling of areas may acquire the necessary flexibility in phase shifts and phase locks noted associated by Thatcher et al. (2016) with higher intelligence. This allows shifting between representations of content and representations of relations so that they may optimally be mapped. Finally, this network may be able to activate a substantiation and evaluation phase that calls upon instances (examples or rules) that allow a closing by a decision. At the mental level, these extra additions may be seen in the symbolic units which are episodic in the first cycle (no involvement of the network above), representational but perceptually-dependent in the second cycle (minimal involvement of the network, with probable dominance of the IPL component), rule-based and language encoded in the third cycle (establishment of the network above, with weak involvement of the rlPFC component), and principle-based and language or arbitrary symbol systems (e.g., mathematical) encoded in the fourth cycle (full activation of the network IFPN). Thus, the expansion of the core executive program of each next cycle in representational scope, procedural flexibility, and cognizance resolution parallels brain network expansion, implying that further representational and inferential possibilities become possible because further brain lines are there to support them.

We view the skeletal executive network as a scaffold for the development of more specialized networks, such as the various logical schemes of deductive reasoning, problem solving strategies in mathematics, moral principles in the social domain, etc. That is, content rich networks are built around the executive scaffold of each phase via a process of translation of the scaffold network into the domainspecific relations and constraints. Building these networks requires the activation of specific circuitry that would carry on the representation of the specific information and relations involved. At both the brain and the mental level, knowledge expansion occurs by adding specific notes peripherally to the main networks. In short, cognitive development involves superimposed sheets of representations in the fashion that brain development involves superimposed sheets of neuronal representations embedded in each other. At the psychological level, higher level representations "are aware" of lower level representations. At the neuronal level, higher level neuronal representations serve as "markers" of lower level representations which may be used to call or activate them, if needed.

We suggested that cognizance rather than speed or working memory is the causal factor for developmental transitions (Demetriou and Spanoudis, 2018). The view of consciousness as a recursive system of interactions between a central executive-selection network and other brain systems renders it central in generating new mental content through its continuous rewiring. Specifically, at any time in development, the content and precision of awareness and cognizance depends on the state, differentiation, and synchronization of globally synchronized networks. Thus, in each developmental phase, the awareness possible is commensurate with the network available. We showed that in each 
next phase increasingly more local networks are hooked onto the global executive network and more long-distance connections are added. This addition goes hand in hand with awareness in each cycle. That is, the resolution and precision of awareness in each cycle reflects the differentiation and tuning of the brain networks involved in consciousness oscillation cycles producing mental units to be availed for attention and executive control. These assumptions may explicate recent strong findings about transfer of cognitive training: transfer may go top-down from relational thought and awareness to attention control and working memory but it does not go bottom-up from these latter processes to fluid intelligence (Christoforides et al. 2016; Papageorgiou et al. 2017; Protzko 2017; Shipstead et al. 2012).

Brain rhythms also change. Overall, with development, activation times drop drastically (e.g., activation time of the ACC, indicated in P300, is $400 \mathrm{msec}$ in children but only 50 msec in adults; Rueda et al. 2005); power increases, but relative power across bands appears to recycle in a fashion reminiscent of mental recycling; cross-band coupling becomes more refined and tuned; networks become more focalized and refined in the fashion that in more intelligent individuals activation is passed on local networks which are precisely modulated by long-distance top-down activation. These changes are reflected in overall changes in processing speed from infancy to adulthoof (Kail, 2000).

However, processing speed and working memory may index cycle and phase transitions in addition to overall development. For instance, when sensorimotor networks are hooked onto a parietal association hub or when, later, this integrated network is hooked onto a pre-frontal hub, sometime is needed to practice and consolidate the new network. Thus, at an initial phase in the functioning of the network, increases in processing speed would reflect changes in the flow of its activation until the core of the network is consolidate. After a certain point in time, the network expands to include already available instances of the lower level networks. At this phase, working memory capacity would be a better psychological marker of network expansion, because it reflects its horizontal expansion and top-down direction in interlinking representations. However, increases in working memory capacity reflect rather than cause increases in the complexity of networks. The findings of Wendelken et al. (2015), indicating that speed was a predictor of change in reasoning at 6-8 years, when dlPFC was dissociated from the vIPFC as a basis of reasoning, and working memory at 8-10 years, when the left and right dlPFC were connected, imply that changes in the rate of change of speed and working memory reflect different phases in the inter-linking between underlying brain networks. These patterns match well with the finding that processing speed is a better index of cognitive changes at the beginning of developmental cycles and working memory at the end (Demetriou et al., 2013, 2014).

\subsection{Unanswered Questions and Predictions}

Obviously, there are many unanswered questions and problems to solve. For example, there is no cognitive function whose corresponding brain structures and networks are fully known. Moreover, we still do not know what is truly general and what is truly specific in both the brain and the mind. Specifically, how much of each general mental function, such as speed, control, or representational capacity, is associated with general brain qualities (i.e., sheer total brain or cortical volume, overall physical state of neurons and neurotransmitters, connectivity, etc.) and how much is accounted for by the fact that particular brain systems (such as the attention or the control networks) are always engaged in cognitive processing? It might be premature to speak about a genetic $\mathrm{g}$ because the genes identified to relate with intelligence accounted for a very small amount of variance in intelligence $(\sim 5 \%)$. However, some genes are associated with various aspects of brain structure and functioning; these, in turn, are associated with individual differences in intelligence and educational attainment. For instance, Sniekers et al. (2017), based on a GWA meta-analysis of 78,308 individuals, identified 15 genomic loci and 40 genes associated with intelligence. These accounted for $4.8 \%$ of the variance in intelligence. These genes were associated with synapse formation, axon guidance in brain development, and regulation of myogenic and neuronal differentiation. Hill et al. (2017) found 107 independent associations for intelligence and increased the number of genes involved in intelligence to 338, predicting $7 \%$ of individual differences in intelligence. However, intelligence is a polygenic trait, making it premature to speak about a brain $g$ because we still do not know what of the brain is common to all mental processes and what is specific. Even if $g$ may be associated with the very flexibility in re-wiring and re-linking networks according to changing needs and experience (Barbey 2018), the specific contributions by 
particular modules of networks at different phases of learning or development is possible. For instance: some aspects of human genetic make-up frame how the brain is formed and functioning; in turn, specific epigenetic events may tune different aspects of the brain to operate high or low, vis-à-vis forthcoming mental challenges.

Also, we still do not know how each of the various networks carry on its own job (e.g., in terms of rhythms), how the networks interact with each other (e.g., by direct structural connections or by functional coordination), and how they are integrated into a final solution behaviorally and subjectively. And, of course, we know very little about how the various types of change in the brain (e.g., myelination, electrical activity, volume, dispersion, activity of neurotransmitters, connectivity, etc.) interact with cognitive developmental changes.

From the developmental point of view, it is worth examining if recycling in the maturation of brain rhythms reflects recycling in mental development. This would involve the re-working of cross-coupling between low band control rhythms such as delta and theta and alpha and beta bands. One might also assume that spurts in synaptic and cortical pruning may be associated with rewiring and re-alignment of nodal and hub links. This rewiring may coincide with phase and cycle changes at the mental level. It would be interesting to search for band hierarchies in brain synchronization matching cognitive developmental hierarchies where earlier mental achievements are integrated in later achievements. One might hypothesize that very low frequency bands (e.g., delta) control a higher frequency band (e.g., theta) which in turn controls high frequency bands (i.e., alpha, beta, and gamma). Resolving these issues would bring us closer to the grand neuro-cognitive developmental theory of intelligence that would integrate brain with functional maps of mental functions into a common landscape. Obviously, this grand theory is still far ahead of us.

Author Contributions: Conceptualization, G.S. and A.D.; writing-original draft preparation, G.S. and A.D.; writing-review and editing, G.S. and A.D. All authors have read and agreed to the published version of the manuscript.", please turn to the CRediT taxonomy for the term explanation. Authorship must be limited to those who have contributed substantially to the work reported.

Funding: Please add: This research received no external funding

Conflicts of Interest: The authors declare no conflict of interest.

\section{References}

Abu-Akel, Ahmad, and Simone Shamay-Tsoory. 2011. Neuroanatomical and neurochemical bases of theory of mind. Neuropsychologia 49: 2971-2984.

Anderson, John R., and Jon M. Fincham. 2014. Extending problem-solving procedures through reflection. Cognitive Psychology 74: 1-34.

Anderson, Michael L., Josh Kinnison, and Luiz Pessoa. 2013. Describing functional diversity of brain regions and brain networks. Neuroimage 73: 50-58.

Anderson, Michael L. 2014. After Phrenology: Neural Reuse and the Interactive Brain, New York: Bradford.

Anobile, Giovanni, Guido Marco Cicchini, and David C. Burr. 2016. Number as a primary perceptual attribute: A review. Perception 45: 5-31.

Baars, Bernard J. 1993. A cognitive theory of consciousness, Cambridge, MA: Cambridge University Press.

Baddeley, Alan. 2012. Working memory: Theories, models, and controversies. Annual Review of Psychology 63: 129.

Baldwin, James M. 1906. Mental development in the child and the race: Methods and processes. Macmillan.

Barbey, Aron K. 2018. Network neuroscience theory of human intelligence. Trends in Cognitive Sciences 22: 8-20. doi.org/10.1016/j.tics.2017.10.001

Barrouillet, Pierre, and Jean-Francois Lecas. 1999. Mental models in conditional reasoning and working memory, Thinking and Reasoning 5: 289-302.

Baumann, Oliver, Edgar Chan, and Jason B. Mattingley. 2012. Distinct neural networks underlie encoding of categorical versus coordinate spatial relations during active navigation. Neuroimage, 60: 1630-1637.

Basten, Ulrike, Kirsten Hilger, and Christian J. Fiebach. 2015. Where smart brains are different: A quantitative meta-analysis of functional and structural brain imaging studies on intelligence. Intelligence, 51: 10-27. 
Benasich, April A., Zhenkun Gou, Naseem Choudhury, and Kenneth D. Harris. 2008. Early cognitive and language skills are linked to resting frontal gamma power across the first 3 years. Behavioural brain research, 195: 215-222.

Blair, Clancy. Clancy. 2006. How similar are fluid cognition and general intelligence? A developmental neuroscience perspective on fluid cognition as an aspect of human cognitive ability. Behavioral and Brain Sciences, 29: 109-125.

Boly, Melanie, Anil K. Seth, Melanie Wilke, Paul Ingmundson, Bernard Baars, Steven Laureys, David Edelman, and Naotsugu Tsuchiya. 2013. Consciousness in humans and non-human animals: recent advances and future directions, Frontiers in Psychology 4: 625. doi.org/10.3389/fpsyg.2013.00625.

Bosseler, Alexis, Samu Taulu, Elina Pihko, Jyrki Mäkelä, Toshiaki Imada, Antti Ahonen, and Patricia Kuhl. 2013. Theta brain rhythms index perceptual narrowing in infant speech perception. Frontiers in Psychology 4: 690. doi: 10.3389/fpsyg.2013.00690.

Brodmann, Korbinian. 1909. Vergleichende Lokalisationslehre der Grosshirnrinde in ihren Prinzipien dargestellt auf Grund des Zellenbaues, Leipzig: Barth.

Buzsáki, György, and Brendon O. Watson. 2012. Brain rhythms and neural syntax: implications for efficient coding of cognitive content and neuropsychiatric disease. Dialogues in clinical neuroscience 14: 345.

Buzsáki, György, Nikos Logothetis, and Wolf Singer. 2013. Scaling brain size, keeping timing: Evolutionary preservation of brain rhythms. Neuron, 80: 751-764.

Cao, Yinan, Christopher Summerfield, Hame Park, Bruno Lucio Giordano, and Christoph Kayser. 2019. Causal inference in the multisensory brain. Neuron 102: 1076-1087.

Carey, Susan. 2009. The Origin of Concepts. New York: Oxford University Press.

Carlén, Marie. 2017. What constitutes the prefrontal cortex? Science 358: 478-482.

Carroll, John B. 1993. Human cognitive abilities: A survey of factor-analytic studies. New York: Cambridge University Press.

Case, Robbie. 1985. Intellectual development: Birth to adulthood. New York: Academic Press.

Case, Robbie. 1992. The mind's staircase: Exploring the conceptual underpinnings of children's thought and knowledge. New York: Psychology Press.

Case, Robbie, Andreas Demetriou, Maria Platsidou, and Smaragda Kazi. 2001. Integrating concepts and tests of intelligence from the differential and developmental traditions. Intelligence 29: 307-336.

Chevalier, Nicolas, and Agnès Blaye. 2016. Metacognitive monitoring of executive control engagement during childhood. Child Development 87: 1264-1276.

Christoforides, Michael, George Spanoudis, and Andreas Demetriou. 2016. Coping with Logical Fallacies: A Developmental Training Program for Learning to Reason. Child Development 87: 1856-1876.

Clarke, Alex, Brooke M. Roberts, and Charan Ranganath. 2018. Neural oscillations during conditional associative learning. NeuroImage 174: 485-493.

Conway, Martin A. Memory and the self. 2005. Journal of memory and language 53: 594-628.

Cowan, Nelson. 2010. The magical mystery four how is working memory capacity limited, and why? Current Directions in psychological science 19: 51-57.

Cowey, Carolyn M. 1996. Hippocampal sclerosis on working memory. Memory 4: 19-30.

Crick, Francis C., and Christof Koch. 2005. What is the function of the claustrum? Philosophical Transactions of the Royal Society B: Biological Sciences 360: 1271-1279.

de Bie, Henrica MA, Maria Boersma, Sofie Adriaanse, Dick J. Veltman, Alle Meije Wink, Stefan D. Roosendaal, Frederik Barkhof et al. 2012, Resting-state networks in awake five- to eight-year old children. Human. Brain Mapping 33: 1189-1201. doi:10.1002/hbm.21280.

Dehaene, Stanislas. 2011. The Number Sense: How the Mind Creates Mathematics. Oxford: Oxford University Press. Dehaene, Stanislas, Hakwan Lau, and Sid Kouider. 2017. What is consciousness, and could machines have it? Science 358: 486-492.

Demetriou, Andreas, and Karin Bakracevic. 2009. Reasoning and self-awareness from adolescence to middle age: Organization and development as a function of education. Learning and Individual Differences 19: 181-194.

Demetriou, Andreas P., Constantinos Christou, George C. Spanoudis, and Maria Platsidou. 2002. The development of mental processing: Efficiency, working memory, and thinking. Monographs of the Society of Research in Child Development 67: 268. 
Demetriou, Andreas, Anastasia Efklides, Maria Platsidou, and Robert L. Campbell. 1993. The architecture and dynamics of developing mind: Experiential structuralism as a frame for unifying cognitive developmental theories. Monographs of the Society for Research in Child Development 58: 234.

Demetriou, Andreas, Samuel Greiff, Nikolaos Makris, George Spanoudis, Rita Panaoura, and Smaragda Kazi. (submitted). Bridging educational priorities with developmental Priorities: Towards a developmental theory of instruction.

Demetriou, Andreas, and Smaragda Kazi. 2006. Self-awareness in g with processing efficiency and reasoning. Intelligence 34: 297-317.

Demetriou, Andreas, Nikolaos Makris, George Spanoudis, Smaragda Kazi, Michael Shayer, and Elena Kazali. 2018. Mapping the Dimensions of General Intelligence: An Integrated Differential-Developmental Theory. Human Development 61: 4-42.

Demetriou, Andreas, and George Spanoudis. 2017. Mind and intelligence: Integrating developmental, psychometric, and cognitive theories of human mind. In Cognitive Abilities and Educational Outcomes. Edited by Monica Rosen, Yang Hansen, Kajsa Wolff. New York: Springer.

Demetriou, Andreas, and George Spanoudis. 2018. Growing minds: A developmental theory of intelligence, brain, and education. London: Routledge.

Demetriou, Andreas, George Spanoudis, Smaragda Kazi, Antigoni Mouyi, Mislav Stjepan Žebec, Elena Kazali, Hudson Golino, Karin Bakracevic, and Michael Shayer. 2017. Developmental differentiation and binding of mental processes with $\mathrm{g}$ through the life-span. Journal of Intelligence 5: 23. doi:10.3390/jintelligence5020023.

Demetriou, Andreas, George Spanoudis, Michael Shayer, Antigoni Mouyi, Smaragda Kazi, and Maria Platsidou. 2013. Cycles in speed-working memory-G relations: Towards a developmental-differential theory of the mind. Intelligence 41: 34-50.

Demetriou, Andreas, George Spanoudis, Michael Shayer, Sanne Van der Ven, Christopher R. Brydges, Evelyn Kroesbergen, Gal Podjarny, and H. Lee Swanson. 2014. Relations between speed, working memory, and intelligence from preschool to adulthood: Structural equation modeling of 15 studies. Intelligence 46: 107121.

Diamond, Adele. 2013. Executive functions. Annual Review of Psychology 64: 135-168.

Dövencioğlu, Dicle, Hiroshi Ban, Andrew J. Schofield, and Andrew E. Welchman. 2013. Perceptual integration for qualitatively different 3-D cues in the human brain. Journal of cognitive neuroscience 25: 1527-1541.

Dumontheil, Iroise. 2014. Development of abstract thinking during childhood and adolescence: The role of rostrolateral prefrontal cortex. Developmental cognitive neuroscience 10: 57-76.

Edelman, G. M., and Guilio Tononi. 2000. A universe of consciousness: how matter becomes imagination. New York: Basic Books.

Eguchi, Akihiro, Samuel A. Neymotin, Greg D. Horwitz, and Thomas D. Albright. 2017. Representation of color. Reference Module in Neuroscience and Biobehavioral Psychology. doi.org/10.1016/B978-0-12-809324-5.02964-3

Epstein, Herman T. 1986. Stages in human development. Developmental Brain Research 30: 114-119.

Fairhall, Scott, L. 2020. Cross recruitment of domain-selective cortical representations enables flexible semantic knowledge. The Journal of Neuroscience, 19, DOI: https://doi.org/10.1523/JNEUROSCI.2224-19.2020

Fedorenko Evelina \& Idan A. Blank. 2020. Broca's area is not a natural kind. Trends in Cognitive Sciences, https://doi.org/10.1016/j.tics.2020.01.001

Fleming, Stephen M., Rimona S. Weil, Zoltan Nagy, Raymond J. Dolan, and Geraint Rees. 2010. Relating Introspective Accuracy to Individual Differences in Brain Structure. Science 329: 1541-1543.

Fodor, Jerry A. 1975. The language of thought. New York: Thomas Y. Crowell.

Fonlupt, Pierre. 2003. Perception and judgement of physical causality involve different brain structures. Cognitive Brain Research 17: 248-254.

Fransson, Peter, Ulrika Åden, Mats Blennow, and Hugo Lagercrantz. 2011. The functional architecture of the infant brain as revealed by resting-state fMRI. Cerebral cortex 21: 145-154.

Friedman, H. R., and Patricia S. Goldman-Rakic. 1988. Activation of the hippocampus and dentate gyrus by working memory: A 2-deoxyglucos study of behaving rhesus monkeys. Journal of Neuroscience 8: 46934706.

Frith, Uta, and Christopher D. Frith. 2003. Development and neurophysiology of mentalizing. Philosophical Transactions of the Royal Society of London. Series B, Biological Sciences 358: 459-473. 
Galaburda, Albert M. 2002. The neuroanatomy of categories. In The languages of the brain. Edited by Albert M. Galaburda, Stephen M. Kosslyn, and Yves Christen. Cambridge, MA: Harvard University Press.

Gauthier, Isabel, Pawel Skudlarski, John C. Gore, and Adam W. Anderson. 2000. Expertise for cars and birds recruits brain areas involved in face recognition. Nature Neuroscience 3: 191.

Gelman, Susan A. 2003. The essential child: Origins of essentialism in everyday thought. New York: Oxford University Press.

Gentner, Dedre. 2005. The development of relational category knowledge. In Building object categories in developmental time Edited by Lisa Gershkoff-Stowe and David H. Rakison. New York: Psychology Press.

Gignac, Gilles E., and Timothy C. Bates. 2017. Brain volume and intelligence: The moderating role of intelligence measurement quality. Intelligence 64: 18-29.

Glasser, Matthew F., Timothy S. Coalson, Emma C. Robinson, Carl D. Hacker, John Harwell, Essa Yacoub, Kamil Ugurbil et al. 2016. A multi-modal parcellation of human cerebral cortex. Nature, 536: 171-178.

Goel, Vinod, Christian Buchel, Chris Frith, and Raymond J. Dolan. 2000. Dissociation of mechanisms underlying syllogistic reasoning. NeuroImage 12: 504-514.

Goel, Vinod, and Raymond J. Dolan. 2000. Anatomical segregation of component processes in an inductive inference task. Journal of Cognitive Neuroscience 12: 110-119.

Goel, Vinod, and Raymond J. Dolan. 2001. Functional neuroanatomy of three-term relational reasoning. Neuropsychologia, 39: 901-909.

Golino, Hudson F., and Andreas Demetriou. 2017. Estimating the dimensionality of intelligence like data using Exploratory Graph Analysis. Intelligence 62: 54-70.

Goriounova, Natalia A., Djai B. Heyer, René Wilbers, Matthijs B. Verhoog, Michele Giugliano, Christophe Verbist, Joshua Obermayer et al. 2018. Large and fast human pyramidal neurons associate with intelligence. Elife 7: e41714.

Gou, Zhenkun, Naseem Choudhury, and April A. Benasich. 2011. Resting frontal gamma power at 16, 24 and 36 months predicts individual differences in language and cognition at 4 and 5 years. Behavioural brain research 220: 263-270.

Gruber, Oliver, and D. Yves von Cramon. 2003. The functional neuroanatomy of human working memory revisited Evidence from 3-T fMRI studies using classical domain-specific interference tasks. NeuroImage 19: 797-809.

Gruber, Oliver, and Thomas Goschke. 2004. Executive control emerging from dynamic interactions between brain systems mediating language, working memory and attentional processes. Acta Psychologica 115: 105-121.

Gustafsson, Jan-Eric. 1984. A unifying model for the structure of intellectual abilities. Intelligence 8: 179-203.

Haier, Richard J. 2016. The neuroscience of intelligence. Cambridge: Cambridge University Press.

Halford, Graeme S., William H. Wilson, and Steven Phillips. 1998. Processing capacity defined by relational complexity: Implications for comparative, developmental, and cognitive psychology. Behavioral and Brain Sciences 21: 803-831.

Harris, Irina M., Gary F. Egan, Cynon Sonkkila, Henri J. Tochon-Danguy, George Paxinos, and John DG Watson. 2000. Selective right parietal lobe activation during mental rotation. Brain 123: 65-73.

Haxby, James V., Leslie G. Ungerleider, Barry Horwitz, Jose Ma Maisog, Stanley I. Rapoport, and Cheryl L. Grady. 1996. Face encoding and recognition in the human brain. Proceedings of the National Academy of Sciences 93: 922-927.

Hermer, Linda, and Elizabeth Spelke. 1996. Modularity and development: the case of spatial reorientation. Cognition 61: 195-232.

Hill, William D., Gail Davies, Andrew M. McIntosh, Catharine R. Gale, and Ian J. Deary. 2017. A combined analysis of genetically correlated traits identifies 107 loci associated with intelligence. BioRxiv 160291. doi: http://dx.doi.org/10.1101/160291.

Hoff, G. E., Martijn Van Den Heuvel, Manon JNL Benders, Karina J. Kersbergen, and Linda S. de Vries. 2013. On development of functional brain connectivity in the young brain. Frontiers in Human Neuroscience 650. doi: 10.3389/fnhum.2013.00650

Holmboe, Karla, Arielle Bonneville-Roussy, Gergely Csibra, and Mark H. Johnson. 2017. Longitudinal development of attention and inhibitory control during the first year of life. Developmental science 21: e12690. 
Hudspeth, William J., and Karl H. Pribram. 1992. Psychophysiological indices of cerebral maturation. International Journal of Psychophysiology 12: 19-29.

Hwang, Kai, Michael N. Hallquist, and Beatriz Luna. 2012. The development of hub architecture in the human functional brain network. Cerebral Cortex 23: 2380-2393. doi:10.1093/cercor/bhs227

Hwang, Kai, Avniel S. Ghuman, Dara S. Manoach, Stephanie R. Jones, and Beatriz Luna. 2016. Frontal preparatory neural oscillations associated with cognitive control: A developmental study comparing young adults and adolescents. NeuroImage 136: 139-148.

Ide, Jaime S., Pradeep Shenoy, J. Yu Angela, and R. Li Chiang-Shan. 2013. Bayesian prediction and evaluation in the anterior cingulate cortex. Journal of Neuroscience 33: 2039-2047.

Irrmischer, Mona, Simon-Shlomo Poil, Huibert D. Mansvelder, Francesca Sangiuliano Intra, and Klaus Linkenkaer-Hansen. 2018. Strong long-range temporal correlations of beta/gamma oscillations are associated with poor sustained visual attention performance. European Journal of Neuroscience 46: 26742683.

Jaušovec, Norbert, and Ksenija Jaušovec. 2014. Increasing working memory capacity with theta transcranial alternating current stimulation (tACS). Biological Psychology 96: 42-47.

Jensen, Arthur R. 1998. Human evolution, behavior, and intelligence. The $g$ factor: The science of mental ability. Westport, CT: Praeger.

Jensen, Ole, and John E. Lisman. 2005. Hippocampal sequence-encoding driven by a cortical multi-item working memory buffer. Trends in Neurosciences 28: 67-72.

Jonides, John, Steven C. Lacey, and Derek Evan Nee. 2005. Processes of working memory in mind and brain. Current Directions of Psychological Science 14: 2-5.

Jung, Rex E., and Richard J. Haier. 2007. The Parieto-Frontal Integration Theory P-FIT of Intelligence: Converging Neuroimaging Evidence. Behavioral and Brain Sciences 30: 135-154.

Kail, Robert V. 2007. Longitudinal evidence that increases in processing speed and working memory enhance children's reasoning. Psychological Science 18: 312.

Kanwisher, Nancy, Josh McDermott, and Marvin M. Chun. 1997. The fusiform face area: a module in human extrastriate cortex specialized for face perception. Journal of Neuroscience 17: 4302-4311.

Kawamichi, Hiroaki, Yoshiaki Kikuchi, Madoka Noriuchi, Atsushi Senoo, and Shoogo Ueno. 2007. Distinct neural correlates underlying two- and three-dimensional mental rotations using three-dimensional objects. Brain Research 1144: 117-126.

Kazi, Smaragda, Elena Kazali, Nikolaos Makris, George Spanoudis, and Andreas Demetriou. 2019. Cognizance in cognitive development: A longitudinal study. Cognitive Development 52: 100805.

Kievit, Rogier A., Simon W. Davis, John Griffiths, Marta M. Correia, and Richard N. Henson. 2016. A watershed model of individual differences in fluid intelligence. Neuropsychologia 91: 186-198.

Klingberg, Torkel. 1998. Concurrent performance of two working memory tasks: Potential mechanisms of interference. Cerebral cortex 8: 593-601.

Kovacs, Kristof, and Andrew RA Conway. 2016. Process overlap theory: A unified account of the general factor of intelligence. Psychological Inquiry 27: 151-177.

Kurth, Salomé, Maya Ringli, Anja Geiger, Monique LeBourgeois, Oskar G. Jenni, and Reto Huber. 2010. Mapping of cortical activity in the first two decades of life: A high-density sleep electroencephalogram study. Journal of Neuroscience 30: 13211-13219.

Kyllonen, Patrick C., and Raymond E. Christal. 1990. Reasoning ability is little more than working memory capacity? Intelligence 14: 389-433.

Lamme, Victor AF. 2006. Towards a true neural stance on consciousness. Trends in cognitive sciences 10: 494-501.

Lisman, John. 2005. T The theta/gamma discrete phase code occuring during the hippocampal phase precession may be a more general brain coding scheme. Hippocampus 15: 913-922.

Lisman, John E., and Ole Jensen. 2013. The Theta-Gamma neural code. Neuron 77: 1002-1016.

Livingstone, Margaret, and David Hubel. 1988. Segregation of form, color, movement, and depth: anatomy, physiology, and perception. Science 240: 740-749.

Mackey, Allyson P., Alison T. Miller Singley, and Silvia A. Bunge. 2013. Intensive reasoning training alters patterns of brain connectivity at rest. Journal of Neuroscience 33: 4796-4803.

Maguire, Mandy J., Matthew R. Brier, and Thomas C. Ferree. 2010. EEG theta and alpha responses reveal qualitative differences in processing taxonomic versus thematic semantic relationships. Brain and language 114: 16-25. 
Mahon, Bradford Z., and Alfonso Caramazza. 2011. What drives the organization of object knowledge in the brain? Trends in cognitive sciences 15: 97-103.

Mahy, Caitlin EV, Louis J. Moses, and Jennifer H. Pfeifer. 2014. How and where: Theory-of-mind in the brain. Developmental cognitive neuroscience 9: 68-81.

Makris, Nikolaos, Dimitrios Tachmatzidis, Andreas Demetriou, and George Spanoudis. 2017. M Mapping the evolving core of intelligence: Changing relations between executive control, reasoning, language, and awareness. Intelligence 62: 12-30.

Marek, Scott, Kai Hwang, William Foran, Michael N. Hallquist, and Beatriz Luna. 2015. The contribution of network organization and integration to the development of cognitive control. PLoS Biology 13: e1002328 DOI:10.1371/journal.pbio.1002328.

Marshall, Peter J., Yair Bar-Haim, and Nathan A. Fox. 2002. Development of the EEG from 5 months to 4 years of age. Clinical Neurophysiology 113: 1199-1208.

Martínez, Kenia, Sarah K. Madsen, Anand A. Joshi, Shantanu H. Joshi, Francisco J. Roman, Julio Villalon-Reina, Miguel Burgaleta et al. 2015. Reproducibility of brain-cognition relationships using three cortical surfacebased protocols: An exhaustive analysis based on cortical thickness. Human brain mapping 36: 3227-3245.

Mashour, George, A., Pieter Roelfsema, Jean-Pierre Changeux, and Stanislas Dehaene. 2020. Conscious processing and the Global Neuronal Workspace Hypothesis. Neuron, 105, https://doi.org/10.1016/j.neuron.2020.01.026

Meeter, M., C. E. Myers, and M. A. Gluck. 2005. Integrating incremental learning and episodic memory models of the hippocampal region. Psychological review 112: 560.

Moreno-Juan, Verónica, Anton Filipchuk, Noelia Antón-Bolanos, Cecilia Mezzera, Henrik Gezelius, Belen Andrés, Luis Rodríguez-Malmierca et al. 2017. Prenatal thalamic waves regulate cortical area size prior to sensory processing. Nature Communications 8: 14172. doi:10.1038/ncomms14172.

Menon, Vinod, and Lucina Q. Uddin. 2010. Saliency, switching, attention and control: a network model of insula function. Brain Structure and Function 214: 655-667.

Millán, Ana P., J. J. Torres, S. Johnson, and J. Marro. 2018. Concurrence of form and function in developing networks and its role in synaptic pruning. Nature communications 9: 1-10.

Miyake, Akira, and Naomi P. Friedman. 2012. The nature and organization of individual differences in executive functions: Four general conclusions. Current Directions in psychological science 21: 8-14.

Moshman, David. 2004. Adolescent rationality and development: Cognition, morality, identity. New York, NY: Psychology Press.

Nieder, Andreas, and Stanislas Dehaene. 2009. Representation of number in the brain. Annual review of neuroscience 32: 185-208.

Orekhova, E. V., T. A. Stroganova, I. N. Posikera, and M. Elam. 2006. EEG theta rhythm in infants and preschool children. Clinical neurophysiology 117: 1047-1062.

Osherson, Daniel, Daniela Perani, Stefano Cappa, Tatiana Schnur, Franco Grassi, and Ferruccio Fazio. 1998. Distinct brain loci in deductive versus probabilistic reasoning. Neuropsychologia 36: 369-376.

Papageorgiou, Eleni, Constantinos Christou, George Spanoudis, and Andreas Demetriou. 2016. Augmenting intelligence: Developmental limits to learning-based cognitive change. Intelligence 56: 16-27.

Patterson, Karalyn, Peter J. Nestor, and Timothy T. Rogers. 2007. Where do you know what you know? The representation of semantic knowledge in the human brain. Nature Reviews Neuroscience 8: 976.

Pascual-Leone, Juan. 1970. A mathematical model for the transition rule in Piaget's developmental stages. Acta psychologica 63: 301-345.

Pennartz, Cyriel MA. 2015. The brain's representational power: on consciousness and the integration of modalities. London: MIT Press.

Peters, Lien, and Bert De Smedt. 2018. Arithmetic in the developing brain: a review of brain imaging studies. Developmental Cognitive Neuroscience, 30: 265-279.

Petersen, Steven E., and Michael I. Posner. 2012. The attention system of the human brain: 20 years after. Annual review of neuroscience 35: 73-89.

Piaget, Jean. 1970. Piaget's theory. In Carmichael's handbook of child development Edited by P. H. Mussen. New York: Wiley.

Piaget, Jean. 2014. Studies in reflecting abstraction. London: Psychology Press.

Piazza, Manuela, Philippe Pinel, Denis Le Bihan, and Stanislas Dehaene. 2007. A magnitude code common to numerosities and number symbols in human intraparietal cortex. Neuron 53: 293-305. 
Posner, Michael I., and Mary K. Rothbart. 2007. Research on attention networks as a model for the integration of psychological science. Annual Review of Psychology 58: 1-23.

Protzko, John. 2015. The environment in raising early intelligence: A meta-analysis of the fadeout effect. Intelligence, 53: 202-210.

Ranganath, Charan, and Mark D'Esposito. 2005. Directing the mind's eye. Prefrontal, inferior, and medial temporal mechanisms for visual working memory. Current opinion in neurobiology 15: 175-182.

Reinhart, Robert MG. 2017. Disruption and rescue of interareal theta phase coupling and adaptive behavior. Proceedings of the National Academy of Sciences 114: 11542-11547.

Repovš, Grega, and Alan Baddeley. 2006. The multi-component model of working memory: explorations in experimental cognitive psychology. Neuroscience 139: 5-21.

Reverberi, Carlo, Doris Pischedda, Michele Burigo, and Paolo Cherubini. Deduction without awareness. Acta psychologica 139: 244-253.

Rolfs, Martin, Michael Dambacher, and Patrick Cavanagh. 2013. Visual adaptation of the perception of causality. Current Biology 23: 250-254.

Roser, Matthew E., Jonathan A. Fugelsang, Kevin N. Dunbar, Paul M. Corballis, and Michael S. Gazzaniga. 2005. Dissociating processes supporting causal perception and causal inference in the brain. Neuropsychology 19: 591.

Rothbart, Mary K., and Michael I. Posner. 2015. The developing brain in a multitasking world. Developmental Review 35: 42-63.

Rodríguez-Martínez, Elena I., Catarina I. Barriga-Paulino, María A. Rojas-Benjumea, and Carlos M. Gómez. 2015. Co-maturation of theta and low-beta rhythms during child development. Brain topography 28: 250-260.

Rounis, Elisabeth, Brian Maniscalco, John C. Rothwell, Richard E. Passingham, and Hakwan Lau. 2010. Thetaburst transcranial magnetic stimulation to the prefrontal cortex impairs metacognitive visual awareness. Cognitive Neuroscience 1: 165-175.

Rubin, Rachael, Hillary Schwarb, Heather Lucas, Michael Dulas, and Neal Cohen. 2017. Dynamic hippocampal and prefrontal contributions to memory processes and representations blur the boundaries of traditional cognitive domains. Brain sciences 7: 82.

Rubinov, Mikail, Olaf Sporns, Cees van Leeuwen, and Michael Breakspear. 2009. Symbiotic relationship between brain structure and dynamics. BMC Neuroscience 10: 55. DOI: 10.1186/1471-2202-10-55.

Rueda, M. Rosario, Michael I. Posner, and Mary K. Rothbart. 2005. The development of executive attention: contributions to the emergence of self-regulation. Developmental Neuropsychology 28: 573-594. doi: 10.1207/s15326942dn2802_2.

Sabbagh, Mark A., Lindsay C. Bowman, Lyndsay E. Evraire, and Jennie MB Ito. 2009. Neurodevelopmental Correlates of Theory of Mind in Preschool Children. Child Development 80: 1147-1162.

Salehi, Mehraveh, Amin Karbasi, Daniel S. Barron, Dustin Scheinost, \& R. Todd Constable. 2020. Individualized functional networks reconfigure with cognitive state. NeuroImage, 206, 116233.

Sauseng, Paul, Birgit Griesmayr, Roman Freunberger, and Wolfgang Klimesch. 2010. Control mechanisms in working memory: A possible function of EEG theta oscillations. Neuroscience $\mathcal{E}$ Biobehavioral Reviews 34: 1015-1022.

Saxe, Rebecca, and Susan Carey. 2006. The perception of causality in infancy. Acta psychologica 123: 144-65.

Scherf, K. Suzanne, John A. Sweeney, and Beatriz Luna. 2006. Brain basis of developmental change in visuospatial working memory. Journal of Cognitive Neuroscience 18: 1045-1058.

Schubert, Anna-Lena, Dirk Hagemann, and Gidon T. Frischkorn. 2017. Is general intelligence little more than the speed of higher-order processing? Journal of Experimental Psychology: General 146: 1498. doi.org/10.1037/xge0000325.

Scott, Ryan B., Zoltan Dienes, Adam B. Barrett, Daniel Bor, and Anil K. Seth. 2014. Blind insight: metacognitive discrimination despite chance task performance. Psychological science 25: 2199-2208.

Segneri, Marco, Hongiie Bi, Simona Olmi, and Alessandro Torcini. 2020. Theta-nested gamma oscillations in next generation neural mass models. arXiv preprint arXiv:2003.04000.

Sergent, Claire, and Lionel Naccache. 2012. Imaging neural signatures of consciousness: 'What', 'When', 'Where' and 'How' does it work? Archives italiennes de biologie 150: 91-106.

Shaw, Philip, Deanna Greenstein, Jason Lerch, Liv Clasen, Rhoshel Lenroot, N. E. E. A. Gogtay, Alan Evans, J. Rapoport, and J. Giedd. 2006. Intellectual ability and cortical development in children and adolescents. Nature 440: 676. 
Siegal, Michael, and Rosemary Varley. 2002. Neural systems involved in 'theory of mind'. Nature Reviews Neuroscience 3: 463.

Siegler, Robert S. 2016. Continuity and change in the field of cognitive development and in the perspectives of one cognitive developmentalist. Child Development Perspectives 10: 128-133.

Shipstead, Zach, Thomas S. Redick, and Randall W. Engle. 2012. Is working memory training effective? Psychological bulletin 138: 628. doi:10.1037/a0027473.

Simion, Francesca, Viola Macchi Cassia, Chiara Turati, and Eloisa Valenza. 2001. The origins of face perception: specific versus non-specific mechanisms. Infant and Child Development: An International Journal of Research and Practice 10: 59 - 65.

Sniekers, Suzanne, Sven Stringer, Kyoko Watanabe, Philip R. Jansen, Jonathan RI Coleman, Eva Krapohl, Erdogan Taskesen et al. 2017. Genome-wide association meta-analysis of 78,308 individuals identifies new loci and genes influencing human intelligence. Nature genetics 49: 1107. doi:10.1038/ng.3869.

Somsen, Riek JM, Ben J. van't Klooster, Maurits W. van der Molen, Harry MP van Leeuwen, and Rob Licht. 1997. Growth spurts in brain maturation during middle childhood as indexed by EEG power spectra. Biological psychology 44: 187-209.

Song, Ming, Yuan Zhou, Jun Li, Yong Liu, Lixia Tian, Chunshui Yu, and Tianzi Jiang. 2008. Brain spontaneous functional connectivity and intelligence. NeuroImage 41: 1168-1176.

Spearman, C. E. 1904. 1904: General intelligence, objectively determined and measured. American Journal of Psychology 15: 201-292.

Sporns, Olaf. 2016. Networks of the Brain. Cambridge, MA: MIT Press.

Sporns, Olaf, Dante R. Chialvo, Marcus Kaiser, and Claus C. Hilgetag. 2004. Organization, development and function of complex brain networks. Trends in cognitive sciences 8: 418-425.

Squire, Larry R. 1992. Memory and the hippocampus: A synthesis from rats, monkeys, and humans. Psychological review 92: 195.

Spanoudis, George, Andreas Demetriou, Smargada Kazi, Katerina Giorgala, and Valentina Zenonos. 2015. Embedding cognizance in intellectual development. Journal of Experimental Child Psychology 132: 32-50.

Stiles, Joan, and Terry L. Jernigan. 2010. The basics of brain development. Neuropsychology review 20: 327-348.

Striedter, George, F. (2005) Principles of brain evolution. Sunderland, MA: Sinauer Associates.

Sun, Chen, Wannan Yang, Jared Martin, and Susumu Tonegawa. 2020. Hippocampal neurons represent events as transferable units of experience. Nature Neuroscience 1-13. https://doi.org/10.1038/s41593-020-0614-x.

Sun, Hua-Chun, Hiroshi Ban, Massimiliano Di Luca, and Andrew E. Welchman. 2015. fMRI evidence for areas that process surface gloss in the human visual cortex. Vision research 109: 149-157.

Supekar, Kaustubh, Lucina Q. Uddin, Katherine Prater, Hitha Amin, Michael D. Greicius, and Vinod Menon. 2010. Development of functional and structural connectivity within the default mode network in young children. NeuroImage 52: 290-301.

Taylor, P., J. N. Hobbs, J. Burroni, and H. T. Siegelmann. 2015. The global landscape of cognition: hierarchical aggregation as an organizational principle of human cortical networks and functions. Scientific reports 5: 18112, DOI: 10.1038/srep18112.

Tenenbaum, Joshua B., Charles Kemp, Thomas L. Griffiths, and Noah D. Goodman. 2011. How to grow a mind: Statistics, structure, and abstraction. Science 331: 1279-1285. doi: 10.1126/science.1192788.

Testolin, Alberto, Youzhi Zou, and James L. McClelland. 2020. Numerosity discrimination in deep neural networks: Initial competence, developmental refinement and experience statistics. Developmental Science https://doi.org/10.1111/desc.12940.

Thatcher, Robert W. 1992. Cyclic cortical reorganization during early childhood. Brain and cognition 20: 24-50.

Thatcher, Robert W. 1994. Cyclic cortical reorganization: Origins of human cognitive development. In Human Behavior and the developing brain Edited by Dawson, Geraldine Ed, and Kurt W. Fischer. New York: The Guilford Press.

Thatcher, R. W., North, D. M., and Biver, C. J. 2005. EEG and intelligence: Relations between EEG coherence, EEG phase delay and power. Clinical Neurophysiology, 116, 2129-2141.

Thatcher, R. W., E. Palmero-Soler, D. M. North, and C. J. Biver. 2016. Intelligence and eeg measures of information flow: efficiency and homeostatic neuroplasticity. Scientific reports 6: 38890.

Thompson, Paul M., Jay N. Giedd, Roger P. Woods, David MacDonald, Alan C. Evans, and Arthur W. Toga. 2000. Growth patterns in the developing brain detected by using continuum tensor maps. Nature 404: 190. 
Thut, Gregor, and Carlo Miniussi. 2009. New insights into rhythmic brain activity from TMS-EEG studies. Trends in cognitive sciences 134: 182-189.

Tran, Randy, and Harold Pashler. 2017. Learning to exploit a hidden predictor in skill acquisition: Tight linkage to conscious awareness. PLoS one 12: e0179386. https://doi.org/10.1371/journal. pone.0179386.

Yoon, Taejib, Jeffrey Okada, Min W. Jung, and Jeansok J. Kim. 2008. Prefrontal cortex and hippocampus subserve different components of working memory in rats. Learning E memory 15: 97-105.

Yue, Qiuhai, Randi C. Martin, Simon Fischer-Baum, Aurora I. Ramos-Nuñez, Fengdan Ye, and Michael W. Deem. 2017. Brain modularity mediates the relation between task complexity and performance. Journal of cognitive neuroscience 29: 1532-1546.

Van Der Maas, Han, Kees-Jan Kan, Maarten Marsman, and Claire E. Stevenson. 2017. Network models for cognitive development and intelligence. Journal of Intelligence 5: 16. doi:10.3390/jintelligence5020016.

Vendetti, Michael S., and Silvia A. Bunge. 2014. Evolutionary and developmental changes in the lateral frontoparietal network: a little goes a long way for higher-level cognition. Neuron 84: 906-917.

Vergauwe, Evie, Egbert Hartstra, Pierre Barrouillet, and Marcel Brass. 2015. Domain-general involvement of the posterior frontolateral cortex in time-based resource-sharing in working memory: An fMRI study. NeuroImage 115: 104-116.

Wang, Yin, Richard Ramsey, and Antonia F. de C. Hamilton. 2011. The control of mimicry by eye contact is mediated by medial prefrontal cortex. Journal of Neuroscience 31: 12001-12010.

Wendelken, Carter, Emilio Ferrer, Kirstie J. Whitaker, and Silvia A. Bunge. 2015. Fronto-Parietal network reconfiguration supports the development of reasoning ability. Cerebral cortex 26: 2178-2190.

Wendelken, Carter, Emilio Ferrer, Simona Ghetti, Stephen K. Bailey, Laurie Cutting, and Silvia A. Bunge. 2017. Frontoparietal structural connectivity in childhood predicts development of functional connectivity and reasoning ability: a large-scale longitudinal investigation. Journal of Neuroscience 37: 8549-8558.

Wiesmann, Charlotte Grosse, Jan Schreiber, Tania Singer, Nikolaus Steinbeis, and Angela D. Friederici. 2017. White matter maturation is associated with the emergence of Theory of Mind in early childhood. Nature communications 8: 14692. DOI: 10.1038/ncomms14692.

Wokke, Martijn E., Dalila Achoui, \& Axel Cleermans. 2020. Action information contributes to metacognitive decision-making. Scientific Reports, 10: 3632, https://doi.org/10.1038/s41598-020-60382-y

$\mathrm{Xu}$, Fei. (2019). Towards a Rational Constructivist Theory of Cognitive Development. Psychological Review http://dx.doi.org/10.1037/rev0000153.

Zamora-López, Gorka, Changsong Zhou, and Jürgen Kurths. 2010. Cortical hubs form a module for multisensory integration on top of the hierarchy of cortical networks. Frontiers in neuroinformatics 4: 1

Zelazo, Philip David. 2015. Executive function: Reflection, iterative reprocessing, complexity, and the developing brain. Developmental Review 38: 55-68.

Zhen, Zonglei, Huizhen Fang, and Jia Liu. 2013. The hierarchical brain network for face recognition. PloS one 8: e59886. doi: 10.1371/journal.pone.0059886.

Zheng, Xuebin, and Jagath C. Rajapakse. 2006. Learning functional structure from fMRI images. NeuroImage 31: 1601-1613.

(C) 2020 by the authors. Submitted for possible open access publication under the terms and conditions of the Creative Commons Attribution CC BY license http://creativecommons.org/licenses/by/4.0/. 